\title{
HHL correlators, orbit averaging and form factors
}

\author{
Zoltan Bajnok, ${ }^{a}$ Romuald A. Janik ${ }^{b}$ and Andrzej Wereszczyński ${ }^{b}$ \\ ${ }^{a}$ MTA Lendület Holographic QFT Group, Wigner Research Centre, \\ H-1525 Budapest 114, P.O.B. 49, Hungary \\ ${ }^{b}$ Institute of Physics, Jagellonian University, \\ ul. Reymonta 4, 30-059 Kraków, Poland \\ E-mail: bajnok.zoltan@wigner.mta.hu, romuald@th.if .uj.edu.pl, \\ wereszcz@th.if.uj.edu.pl
}

ABSTRACT: We argue that the conventional method to calculate the OPE coefficients in the strong coupling limit for heavy-heavy-light operators in the $\mathcal{N}=4$ Super-Yang-Mills theory has to be modified by integrating the light vertex operator not only over a single string worldsheet but also over the moduli space of classical solutions corresponding to the heavy states. This reflects the fact that we are primarily interested in energy eigenstates and not coherent states. We tested our prescription for the BMN vacuum correlator, for folded strings on $S^{5}$ and for two-particle states. Our prescription for two-particle states with the dilaton leads to a volume dependence which matches exactly to the structure of finite volume diagonal formfactors. As the volume depence does not rely on the particular light operator we conjecture that symmetric OPE coefficients can be described for any coupling by finite volume diagonal form factors.

KEYWORDS: Supersymmetric gauge theory, AdS-CFT Correspondence, Integrable Field Theories

ARXIV EPRINT: 1404.4556 


\section{Contents}

1 Introduction 1

2 Classical limit of quantum expectation values and classical solutions 3

3 Coherent states vs. energy eigenstates 5

4 HHL correlation functions $\quad 6$

5 Three examples $\quad 8$

5.1 The BMN vacuum correlator $\quad 8$

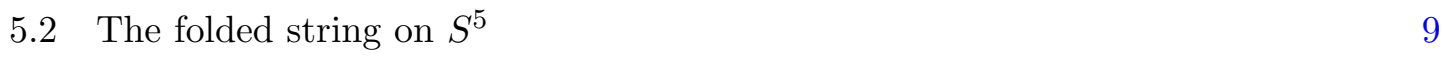

$\begin{array}{ll}5.3 \text { A two-magnon solution in finite volume } & 11\end{array}$

6 Symmetric OPE coefficients and diagonal formfactors 17

$\begin{array}{lll}7 & \text { A conjecture } & 20\end{array}$

8 Conclusions $\quad 20$

A Finite volume diagonal form factors $\quad 22$

A.1 One particle diagonal matrix elements 22

A.2 Two particle diagonal matrix elements 23

A.3 Form factors of special operators 25

\section{Introduction}

The integrability of string theory in $\mathrm{AdS}_{5} \times S^{5}$ spacetime opens up the fascinating possibility of exactly solving a nontrivial interacting gauge theory - the supersymmetric $\mathcal{N}=4$ Super-Yang-Mills, through the use of the AdS/CFT correspondence [1]-[3].

Currently, we have a very detailed and refined understanding of the spectral problem in $\mathcal{N}=4 \mathrm{SYM}$, i.e. the structure of the anomalous dimensions of local gauge invariant operators in the planar limit for, in principle, arbitrary value of the coupling constant [4][18]. The key ingredient exploited here was the translation of this problem into the problem of determining the energy levels of the integrable 2-dimensional worldsheet quantum field theory of the string in $\mathrm{AdS}_{5} \times S^{5}$. The spectral problem for this integrable QFT, although exhibiting numerous novel features, could be solved by following the main steps appearing when solving analogous problems for relativistic integrable field theories, although historically this did not happen exactly in this way, and the current most refined finite reformulation of the exact infinite set of Thermodynamic Bethe Ansatz equations [14]-[18] — the so-called Quantum Spectral Curve [19] does not have an analog in the conventional 
relativistic setting. However, the relativistic integrable QFT could (and did) serve as a guideline for the much more complicated $\mathrm{AdS}_{5} \times S^{5}$ spectral problem.

The second essential ingredient of a 'solution' of a conformal field theory, of which $\mathcal{N}=4 \mathrm{SYM}$ is an example, is the determination of the OPE coefficients of local operators. On the string side the OPE coefficients arise from string interactions and thus worldsheets with 3 boundaries/asymptotic regions and basically we do not have any relativistic integrable QFT setting to act as a guideline. Thus, obtaining exact answers valid at any coupling is extremely challenging.

Despite that, significant progress has been obtained both at weak coupling [20]-[29], and at strong coupling.

In the latter case, it is fruitful to classify gauge theory operators into three broad groups: Heavy operators, which correspond on the string side to classical string solutions, Light operators which are typically protected and correspond to the supergravity modes and Medium operators which correspond to short (massive) string states - the key example being the Konishi operator.

Recent work concentrated either on the case of three heavy operators involving algebraic curve constructions/Pohlmeyer reductions [30]-[33] and [34, 35], three medium or light operators using vertex operators and local flat space approximations [36]-[37] and two heavy and one light case [38]-[57] (HHL correlators). It is this last case which is at the focus of the present paper.

The motivation for this paper, however, goes beyond just the strong coupling limit and involves the search for a framework which would naturally allow for treatment at any coupling. The most general framework for OPE coefficients would most probably be (light cone) string field theory (SFT) [35, 58-60] (and numerous papers in the pp-wave era, in particular [61-65]) as generically the sizes of the three strings would be different. However it has been suggested $[66,67]$ that a simpler framework might be the formfactor formalism, where the emission of the third string would be described in terms of a vertex operator insertion on the worldsheet.

Although, the formfactor approach cannot certainly describe ${ }^{1}$ generic OPE coefficients as the sizes of all the cylinders are distinct, it may be a good description of the case when the third operator does not carry any $J$ charge. In particular, the case of symmetric OPE's, where two of the three operators are identical/conjugate may be describable within this framework. Let us note that, at the same time, this case is an extremely degenerate limit of the potential string field theory description so the formfactor approach may be seen not as an alternative to SFT but rather as a complement.

The formfactor approach in integrable relativistic field theories rapidly becomes more and more complicated when the number of particles becomes large. For this reason the initial motivation for this paper was to compute HHL OPE coefficients where the heavy state is a classical two-particle state with $\mathcal{O}(1)$ momenta. This is quite in contrast to conventional spinning strings which are multiparticle states with constitutents with momenta of order $\mathcal{O}(1 / \sqrt{\lambda})$.

\footnotetext{
${ }^{1}$ At least as long as we remain within the context of the integrable worlsheet QFT of the $\operatorname{AdS}_{5} \times S^{5}$ string quantized in uniform light-cone gauge.
} 
However when investigating this case, we encountered a problem with the commonly accepted prescription for computing HHL correlators. The HHL prescription amounts to integrating the vertex operator of the light state over the classical solution corresponding to a 2-point correlation function of the Heavy operators.

The problem stems from the fact that there is always at least a 1-parameter family of distinct classical solutions corresponding to the 2-point correlation function, namely

$$
z(\tau)=\frac{R}{\cosh \kappa \tau} \quad x(\tau)=x_{0}+R \tanh \kappa \tau \quad \text { and } \quad X^{I}\left(\sigma, \tau-\tau_{0}\right)
$$

for arbitrary $\tau_{0}$, and the result of the standard HHL prescription depends on the value of $\tau_{0}$.

The goal of this paper is twofold. Firstly, we show how to modify the HHL prescription in order to overcome this problem, and moreover, we argue that this is a very general feature of the classical computation of quantum expectation values in states with definite energy. In addition, we argue that at least in the OPE context, the conventional use of coherent states may be inappropriate.

Secondly, we compute, using the modified HHL prescription, the OPE of a two-particle state with a dilaton/lagrangian density and argue that the obtained dependence on the size of the cylinder is exactly of the structure expected for finite volume diagonal formfactors.

The plan of this paper is as follows. In section 2 we review the classical computation of quantum expectation values in some simple quantum-mechanical systems. This brings us to a comparision between coherent states and energy eigenstates in the classical context. Then we move on to describe the modified HHL prescription in section 4, and, in the following section, give three example computations: for the BMN vacuum correlator, the folded $S^{5}$ string and the two-particle classical solution. In section 6 , we compare the structural properties of the answer for two-particle states with the expectations from finite volume diagonal formfactor approach. In section 7 we formulate a conjecture for the structure of symmetric OPE coefficients and close the paper with conclusions. In an appendix we give a brief self-contained review of diagonal finite volume form factors.

\section{Classical limit of quantum expectation values and classical solutions}

Suppose that we are interested in computing a quantum-mechanical expectation value of some operator $f(\hat{x})$ evaluated at time $t$ in a state with definite energy

$$
\langle E|f(\hat{x})(t)| E\rangle \equiv\left\langle\Psi_{E}(t)|f(\hat{x})| \Psi_{E}(t)\right\rangle
$$

We will assume that the energy $E$ is large enough so that the relevant state is semi-classical. Clearly the above expectation value is $t$-independent. We would like to compute the above expression in terms of classical solutions (of the corresponding classical system) with energy $E$ (see [69]). In the following it is important to note that we have always a (rather trivial) family of classical solutions with energy $E$ :

$$
x(t)=x_{\mathrm{cl}}\left(t-t_{0}\right)
$$

where $t_{0}$ takes values within the period of the classical solution $x_{\mathrm{cl}}(t)$.

Let us consider three simple examples. 
1D harmonic oscillator. The wavefunction for the $n^{\text {th }}$ eigenstate is

$$
\Psi_{n}(x)=\frac{1}{2^{n} n !}\left(\frac{m \omega}{\pi \hbar}\right)^{\frac{1}{4}} e^{-\frac{m \omega x^{2}}{2 \hbar}} H_{n}\left(\sqrt{\frac{m \omega}{\hbar}} x\right)
$$

We are interested in states with $n=E / \hbar \omega$ and we take the limit $\hbar \rightarrow 0$ with $E$ fixed. Then the wavefunction becomes

$$
\Psi_{E}(x) \sim \sqrt{\frac{2}{\pi}}\left(A^{2}-x^{2}\right)^{-\frac{1}{4}} \cos \left(\frac{\sqrt{2 E m}}{\hbar} x-n \frac{\pi}{2}\right)
$$

where $A=\sqrt{2 E / m \omega^{2}}$ is the amplitude of the corresponding classical motion. Now taking $\hbar \rightarrow 0$ we get the following formula for the expectation value

$$
\langle E|f(\hat{x})(t)| E\rangle=\frac{1}{\pi} \int_{-A}^{A} \frac{f(x) d x}{\sqrt{A^{2}-x^{2}}}
$$

It is instructive to change variables in the above integral from $x$ to $t_{0}$ through $x=$ $x_{\mathrm{cl}}\left(t-t_{0}\right)=A \sin \omega\left(t-t_{0}\right)$. Then the above expectation value may be rewritten as

$$
\langle E|f(\hat{x})(t)| E\rangle=\frac{1}{T_{0}} \int_{-T_{0} / 2}^{T_{0} / 2} f\left(x_{\mathrm{cl}}\left(t-t_{0}\right)\right) d t_{0}
$$

where $T_{0}=2 \pi / \omega$ is the period. We see that the quantum mechanical expectation value is realized on the classical level through a temporal integral with uniform measure over the family of classical trajectories (2.2), or equivalently over the relevant periodic orbit.

Periodic motion in an arbitrary 1D potential. The above simple formula (2.6) applies without change for an arbitrary 1D potential. Indeed let us take the WKB wavefunction with turning points $x_{0}$ and $x_{1}$ :

$$
\Psi_{\mathrm{WKB}}(x)=\frac{c}{\sqrt{p(x)}} \cos \left(\frac{1}{\hbar} \int p d x\right)
$$

The normalization coefficient can be computed explicitly from

$$
1=\int_{x_{0}}^{x_{1}} \frac{1}{2} \frac{c^{2}}{|p|} d x=\int_{x_{0}}^{x_{1}} \frac{1}{2} \frac{c^{2}}{m\left|\frac{d x}{d t}\right|} d x=\frac{1}{4} \frac{c^{2}}{m} T_{0}
$$

Here $T_{0}$ is the period of the classical motion. Hence we have effectively (as long as we are interested only in position observables)

$$
\left|\Psi_{\mathrm{WKB}}(x)\right|^{2} \sim \frac{2}{T_{0}} \frac{1}{\left|v_{\mathrm{cl}}\right|}
$$

which allows us to change from a spatial to a temporal integral. Hence the quantum mechanical expectation value in the classical limit becomes again

$$
\langle E|f(\hat{x})(t)| E\rangle=\frac{1}{T_{0}} \int_{-T_{0} / 2}^{T_{0} / 2} f\left(x_{\mathrm{cl}}\left(t-t_{0}\right)\right) d t_{0}
$$

exactly as for the harmonic oscillator considered before. 
2D harmonic oscillator. Let us finally consider a 2-dimensional harmonic oscillator, in general with incommensurable periods. Two new features will appear in this case. Firstly, the overall solution is no longer periodic and secondly, the moduli space of relevant classical solutions becomes 2-dimensional. Indeed, the space of classical solutions corresponding to the quantum state $\left|E_{0}, E_{1}\right\rangle$ is now parametrized by two independent shifts:

$$
x(t)=x_{\mathrm{cl}}\left(t-t_{0}\right)=A_{0} \sin \omega_{0}\left(t-t_{0}\right) \quad y(t)=y_{\mathrm{cl}}\left(t-t_{1}\right)=A_{1} \sin \omega_{1}\left(t-t_{1}\right)
$$

In order to overcome the first limitation, i.e. the lack of a common overall period, let us note that we may write (2.6) without an explicit reference to the specific value of the period through the substitution

$$
\frac{1}{T_{0}} \int_{-T_{0} / 2}^{T_{0} / 2} d t_{0} \longrightarrow \lim _{T \rightarrow \infty} \frac{1}{T} \int_{-T / 2}^{T / 2} d t_{0}
$$

Now it is immediate to use the preceeding results and obtain

$$
\left\langle E_{0}, E_{1}\left|\hat{x}^{n} \hat{y}^{m}\right| E_{0}, E_{1}\right\rangle=\lim _{T \rightarrow \infty} \frac{1}{T^{2}} \int_{-\frac{T}{2}}^{\frac{T}{2}} d t_{0} \int_{-\frac{T}{2}}^{\frac{T}{2}} d t_{1}\left(x_{\mathrm{cl}}\left(t-t_{0}\right)\right)^{n}\left(y_{\mathrm{cl}}\left(t-t_{1}\right)\right)^{m}
$$

A generic observable $f(\hat{x}, \hat{y})$ follows by expansion in a power series.

To summarize, we see that a stationary state of given energy (and possibly other commuting quantum numbers) corresponds on the classical level to a moduli space of classical solutions parametrized by time shifts, while quantum expectation values are related to (temporal) averaging of the observable over this moduli space.

\section{Coherent states vs. energy eigenstates}

The above description is very much at odds (but of course not in contradiction!) with the most commonly used way of looking at the classical limit in quantum mechanics, where we typically use the notion of a coherent state. However the difference lies in quite different physical questions that we are addressing. In the case of a coherent state, what we seek is a quantum description of a single given classical trajectory - so that quantum expectation values follow the given classical trajectory e.g.

$$
\left\langle\Psi_{\mathrm{coh}}(t)|f(\hat{x})| \Psi_{\mathrm{coh}}(t)\right\rangle \sim f\left(x_{\mathrm{cl}}(t)\right)
$$

The physical question addressed in the previous section deals, on the other hand, with a classical description of a stationary quantum state in some given energy level. Then the corresponding expectation value is clearly time independent

$$
\left\langle\Psi_{E}(t)|f(\hat{x})| \Psi_{E}(t)\right\rangle \sim \text { const }
$$

and the considerations reviewed in the previous section indicate that the constant may be evaluated through an integral over the moduli space of classical solutions with the given energy (and possibly other quantum numbers characterizing the given quantum state). 
Thus the question which picture to use really depends on whether we are focusing on a given classical trajectory, or rather on a given quantum state with fixed energy. In the case of correlation functions in AdS/CFT the relevant picture is in fact clearly the latter one. Then following the arguments of the previous section, the dual description should really be the full moduli space of appropriate classical solutions and not a single representative.

Nevertheless, for questions related e.g. to the spectral problem, a single representative is clearly sufficient to obtain all information about the energy and charges of the corresponding quantum state. ${ }^{2}$ The same conclusion holds for 2-point correlation functions. We will show, however, that in the case of 3-point correlation functions the treatment of the full moduli space is in fact neccessary.

\section{HHL correlation functions}

In two very important papers [38, 39], a proposal was formulated for computing 3-point correlation functions in the case when two operators are Heavy (i.e. being described by a classical string solution) and almost identical, while the third operator is Light and is described by a supergravity field (or equivalently by an appropriate $\mathcal{O}(1)$ vertex operator on the string worldsheet [40]).

The proposal amounts to integrating the light vertex operator over the classical solution corresponding to the 'heavy' operators. The resulting expression has the form

$$
C_{\mathrm{HHL}}=\mathrm{const} \cdot \int d \tau \int d \sigma V_{L}\left[x_{H}(\sigma, \tau), z_{H}(\sigma, \tau), X_{H}^{I}(\sigma, \tau)\right]
$$

where $x_{H}, z_{H}$ is the AdS part of the classical solution of the heavy state ${ }^{3}$ and $X_{H}^{I}$ is the $S^{5}$ part. The relevant part of the AdS metric is $d s^{2}=z^{-2}\left(d z^{2}+d x^{2}\right) . V_{L}$ is the vertex operator of the light state. E.g. for the dilaton it is given by

$$
V_{L}^{\text {dil }}=\left(\frac{\left(x-x_{0}\right)^{2}+z^{2}}{z}\right)^{-4}\left[\frac{\partial x \bar{\partial} x+\partial z \bar{\partial} z}{z^{2}}+\partial X^{K} \bar{\partial} X^{K}\right]
$$

while for the BMN vacuum $\operatorname{tr} Z^{k}$ it takes the form

$$
V_{L}^{B M N}=\left(\frac{\left(x-x_{0}\right)^{2}+z^{2}}{z}\right)^{-k}\left(X_{1}+i X_{2}\right)^{k}\left[\frac{\partial x \bar{\partial} x-\partial z \bar{\partial} z}{z^{2}}-\partial X^{K} \bar{\partial} X^{K}\right]
$$

where $x_{0}$ is usually taken to 0 . In the above formula (4.1), there is no contribution of the Heavy vertex operators as it was argued that this contribution should cancel with analogous contributions in a 2-point function when we express the OPE coefficient as a ratio of a 3point correlation function and 2-point correlation functions. This cancellation could indeed be expected if there were just a single heavy state classical solution contributing to (4.1).

The discussion in the preceeding sections suggests, however, that we should be dealing with a family of classical solutions corresponding to the heavy state.

\footnotetext{
${ }^{2}$ Note however that integration over collective coordinates was used in [34] to show the cancellation of AdS volume with $\mathrm{SL}(2, C)$ volume to get a finite result for the string computation.

${ }^{3}$ This is a Wick rotated solution with Euclidean worldsheet signature.
} 
Indeed, for heavy operators with nontrivial charges only on the $S^{5}$, there is always at least a 1-dimensional family of relevant classical solutions:

$$
x=R \tanh \kappa\left(\tau-\tau_{0}\right) \quad z=\frac{R}{\cosh \kappa\left(\tau-\tau_{0}\right)} \quad \text { and } \quad X^{I}(\sigma, \tau)
$$

Note that for each $\tau_{0}$ this is a distinct solution as we perform the shift by $\tau_{0}$ only in the AdS part of the solution. ${ }^{4}$ Moreover, there may be additional moduli coming from the $S^{5}$ part of the solution. E.g. in the case of finite-gap solutions, the motion occurs on a $g+1$-dimensional torus, and one is free to consider shifts in all the relevant angle variables.

Below we will write explicit formulas incorporating just the shift in (4.4). The considerations in preceeding sections suggest that we should average over $\tau_{0}$ with uniform measure. We are thus led to

$$
C_{\mathrm{HHL}}=\text { const } \cdot \lim _{T \rightarrow \infty} \frac{1}{T} \int_{-T / 2}^{T / 2} d \tau_{0} \int d \tau \int d \sigma V_{L}\left[x_{H}\left(\sigma, \tau-\tau_{0}\right), z_{H}\left(\sigma, \tau-\tau_{0}\right), X_{H}^{I}(\sigma, \tau)\right]
$$

However this is not the whole story. Now, since we do not have a single saddle point but rather a moduli space of saddle points parametrized by $\tau_{0}$, the contribution of the Heavy vertex operators will not appear just as an overall factor and it will not cancel completely with the one in 2-point functions.

In general we do not know the form of arbitrary vertex operators for classical solutions. However, for operators with all charges on the $S^{5}$, it seems that there is always a universal relevant piece of the form

$$
\left(\frac{(x \pm R)^{2}+z^{2}}{z}\right)^{-\Delta} \longrightarrow(2 R)^{-\Delta} e^{\mp \Delta \kappa\left(\tau-\tau_{0}\right)}
$$

depending on the insertion point of the heavy operator which is either at $x=-R$ or $x=+R$. Now suppose that we regularize our worldsheet to extend from $-\tau_{\max }$ to $\tau_{\max }$. Performing the shift by $\tau_{0}$ will yield the following modifications w.r.t. the same vertex operators evaluated on the unshifted solution:

$$
\begin{aligned}
(2 R)^{-\Delta} e^{-\Delta \kappa\left(-\tau_{\max }-\tau_{0}\right)} & \sim(2 R)^{-\Delta} e^{\Delta \kappa \tau_{\max }} e^{\kappa \Delta \cdot \tau_{0}} \\
(2 R)^{-\Delta} e^{\Delta \kappa\left(\tau_{\max }-\tau_{0}\right)} & \sim(2 R)^{-\Delta} e^{\Delta \kappa \tau_{\max }} e^{-\kappa \Delta \cdot \tau_{0}}
\end{aligned}
$$

So we get an additional factor

$$
e^{-\left(\Delta_{+\infty}-\Delta_{-\infty}\right) \kappa \tau_{0}}
$$

which has to be included in (4.5). So the modified prescription should be

$$
\text { const } \cdot \lim _{T \rightarrow \infty} \frac{1}{T} \int_{-T / 2}^{T / 2} d \tau_{0} \int d^{2} \sigma V_{L}\left[x_{H}\left(\sigma, \tau-\tau_{0}\right), z_{H}\left(\sigma, \tau-\tau_{0}\right), X_{H}^{I}(\sigma, \tau)\right] e^{-\left(\Delta_{+\infty}-\Delta_{-\infty}\right) \kappa \tau_{0}}
$$

Note that in the case that the classical string solution has a higher dimensional moduli space on the $S^{5}$, we would need to include similar factors from the nontrivial $S^{5}$ parts of

\footnotetext{
${ }^{4}$ Of course we could have equivalently made the shift on the $S^{5}$ part.
} 
the unknown classical vertex operators of the heavy states. Similarly, for operators with spin in AdS, the contribution of the heavy vertex operator has to be worked out case by case. Unfortunately, we know the explicit form of the classical vertex operators only in a few cases like the GKP string or a folded string with $S, J \neq 0$ [40]. Then the heavy vertex correction factor (4.9) would have to be modified by terms involving the difference in the spin between the initial and final heavy state. Unfortunately, currently we do not have control over the generic finite-gap solution.

An important case when we may probably sidestep this issue is when the light operator does not carry any conserved charges and the two heavy operators are identical. We will call these OPE coefficients symmetric OPE's and consider them in more detail in the final part of the paper.

\section{$5 \quad$ Three examples}

In this section we will consider three examples involving the use of the modified HHL prescription (4.10). Two of these examples involve spinning strings [38], while the third one involves a classical two-magnon state. For the former case we find that, even though the light vertex operator evaluated on the classical solution depends nontrivially on $\tau_{0}$, the contribution of the heavy vertex operators ${ }^{5}$ cancels this $\tau_{0}$ dependence and we recover previous results from the literature. On the other hand, for the two-magnon computation the averaging over $\tau_{0}$ is absolutely crucial in order to obtain the correct result.

\subsection{The BMN vacuum correlator}

Let us consider the BPS correlator $\left\langle\operatorname{tr} Z^{J} \operatorname{tr} Z^{k} \operatorname{tr} \bar{Z}^{J+k}\right\rangle$ with $J \in \mathcal{O}(\sqrt{\lambda})$ and $k \in \mathcal{O}(1)$. The classical solution on $S^{5}$ is given by $\phi_{1}=i \kappa \tau$ and $\phi_{2}=\frac{\pi}{2}$. Here, we treat $\operatorname{tr} \bar{Z}^{J+k}$ as a 'creation operator' for the string which means that the ingoing $(\tau \rightarrow-\infty)$ heavy string configuration caries $\Delta_{-\infty}=J+k$ scaling dimension. The BMN vacuum vertex operator is given by (4.3)

$$
V_{L}^{\mathrm{BMN}}=\left(\frac{\left(x-x_{0}\right)^{2}+z^{2}}{z}\right)^{-k}\left(X_{1}+i X_{2}\right)^{k}\left[\frac{\partial x \bar{\partial} x-\partial z \bar{\partial} z}{z^{2}}-\partial X^{K} \bar{\partial} X^{K}\right]
$$

Here

$$
\frac{\partial x \bar{\partial} x-\partial z \bar{\partial} z}{z^{2}}=\kappa^{2}\left[\frac{2}{\cosh ^{2} \kappa\left(\tau-\tau_{0}\right)}-1\right] \quad \partial X^{K} \bar{\partial} X^{K}=-\kappa^{2}
$$

Hence the vertex operator evaluated on the solution (4.4) takes the form

$$
\int d \tau \int_{0}^{2 \pi} d \sigma \frac{e^{-k \kappa \tau}}{\cosh ^{2+k} \kappa\left(\tau-\tau_{0}\right)}
$$

As it stands, we see a clear dependence of the formula on the value of the shift parameter $\tau_{0}$. We should now add the contribution from the modification of the heavy vertex operators.

\footnotetext{
${ }^{5}$ At least to the level that we control it.
} 
Since the intermediate state carries very small charges, the difference in the energies of the heavy states appearing in formula (4.10) can be computed using derivatives

$$
-\left(\Delta_{+\infty}-\Delta_{-\infty}\right) \kappa \tau_{0} \quad \longrightarrow \quad \frac{\partial \Delta(J)}{\partial J} k \kappa \tau_{0}
$$

Hence we will get an additional contribution $e^{k \kappa \tau_{0}}$ which transforms (5.3) into

$$
\int d \tau \int_{0}^{2 \pi} d \sigma \frac{e^{-k \kappa\left(\tau-\tau_{0}\right)}}{\cosh ^{2+k} \kappa\left(\tau-\tau_{0}\right)}
$$

Now we can redefine the $\tau$ integral by $\tau_{0}$, which then coincides with the integral in [38]. The leftover $\tau_{0}$ averaging trivializes.

$$
\lim _{T \rightarrow \infty} \frac{1}{T} \int_{-T / 2}^{T / 2} d \tau_{0}=1
$$

\subsection{The folded string on $S^{5}$}

The folded string solution on $S^{5}$ provides for us a more nontrivial example. Firstly it is a genus 1 solution, so we may expect a higher dimensional moduli of classical solutions. Secondly, the dependence of the anomalous dimension on the charges is now much more complicated.

The nontrivial $S^{5}$ part of the solution is given by

$$
\phi_{1}=i w_{1} \tau, \quad \phi_{2}=i w_{2} \tau, \quad \psi=\psi(\sigma)
$$

where

$$
\psi^{\prime 2}+w_{1}^{2} \cos ^{2} \psi+w_{2}^{2} \sin ^{2} \psi=\kappa^{2}
$$

The conserved charges are

$$
\Delta=\sqrt{\lambda} \kappa, \quad J_{1}=\sqrt{\lambda} w_{1} \int_{0}^{2 \pi} \frac{d \sigma}{2 \pi} \cos ^{2} \psi, \quad J_{2}=\sqrt{\lambda} w_{2} \int_{0}^{2 \pi} \frac{d \sigma}{2 \pi} \sin ^{2} \psi
$$

Hence,

$$
J_{1}=\sqrt{\lambda} w_{1} \frac{E(s)}{K(s)}, \quad J_{2}=\sqrt{\lambda} w_{2}\left(1-\frac{E(s)}{K(s)}\right)
$$

where

$$
s \equiv \frac{\kappa^{2}-w_{1}^{2}}{w_{2}^{2}-w_{1}^{2}}, \quad \sqrt{w_{2}^{2}-w_{1}^{2}}=\frac{2}{\pi} K(s)
$$

where the last formula comes from the periodicity of the solution. The dimension $\Delta$ can be (implicitly) expressed in terms of $J_{1}, J_{2}$ as

$$
\begin{array}{r}
\left(\frac{\Delta}{K(s)}\right)^{2}-\left(\frac{J_{1}}{E(s)}\right)^{2}=\frac{4 \lambda}{\pi^{2}} s \\
\left(\frac{J_{2}}{K(s)-E(s)}\right)^{2}-\left(\frac{J_{1}}{E(s)}\right)^{2}=\frac{4 \lambda}{\pi^{2}}
\end{array}
$$


The solution (5.7) allows for independent shifts of $\phi_{1}$ and $\phi_{2}$. We will consider the light vertex operator to again correspond to the BMN vacuum with charge $J_{1}=k$. Hence the angular coordinate $\phi_{2}$ will not appear explicitly (i.e. without an accompanying $\tau$ derivative) in the integrand of the light vertex operator. Also the two heavy states will have exactly the same value of the charge $J_{2}$. This suggests that the contribution of the shift of $\phi_{2}=$ $i w_{2} \tau \rightarrow i w_{2}\left(\tau-\tau_{2}\right)$ will cancel between the two heavy vertex operators. ${ }^{6}$

As before, we will trade the overall shift on the $S^{5}$ for a shift on the AdS part of the solution. The light vertex operator integral then takes the form

$$
\int_{-\infty}^{\infty} \frac{d \tau}{\cosh ^{k} \kappa\left(\tau-\tau_{0}\right)} \int_{0}^{2 \pi} d \sigma e^{-w_{1} k \tau} \sin ^{k} \psi\left[\kappa^{2} \tanh ^{2} \kappa\left(\tau-\tau_{0}\right)+\left(\frac{\partial \vec{n}}{\partial \tau}\right)^{2}\right]
$$

where $\left(\partial_{\tau} \vec{n}\right)^{2}$ is $\tau$ independent.

We now have to evaluate the contribution coming from the heavy vertex operators (again the barred operator with $\Delta=\Delta\left(J_{1}+k, J_{2}\right)$ is put at $\tau=-\infty$ ). The contribution is given by

$$
e^{-\left(\Delta_{+\infty}-\Delta_{-\infty}\right) \kappa \tau_{0}}=e^{\frac{\partial \Delta\left(J_{1}, J_{2}\right)}{\partial J_{1}} k \kappa \tau_{0}}
$$

Due to the implicit dependence of the energy on the conserved charges, the computation of the derivative

$$
\frac{\partial \Delta\left(J_{1}, J_{2}\right)}{\partial J_{1}}
$$

is much more involved. First we act with $\partial / \partial J_{1}$ on $(5.12),(5.13)$ :

$$
\begin{aligned}
\frac{\Delta}{K^{2}(s)} \Delta_{J_{1}}-\frac{\Delta^{2}}{K^{3}(s)} K^{\prime}(s) \frac{\partial s}{\partial J_{1}}-\frac{J_{1}}{E^{2}(s)}+\frac{J_{1}^{2}}{E^{3}(s)} E^{\prime}(s) \frac{\partial s}{\partial J_{1}} & =\frac{2 \lambda}{\pi^{2}} \frac{\partial s}{\partial J_{1}} \\
-\frac{J_{2}^{2}}{(K(s)-E(s))^{3}}\left(K^{\prime}(s)-E^{\prime}(s)\right) \frac{\partial s}{\partial J_{1}}-\frac{J_{1}}{E^{2}(s)}+\frac{J_{1}^{2}}{E^{3}(s)} E^{\prime}(s) \frac{\partial s}{\partial J_{1}} & =0
\end{aligned}
$$

From the last equation we find

$$
\frac{\partial s}{\partial J_{1}}=\frac{J_{1}}{E^{2}(s)\left(\frac{J_{1}^{2} E^{\prime}(s)}{E^{3}(s)}-\frac{J_{2}^{2}}{(K(s)-E(s))^{3}}\left(K^{\prime}(s)-E^{\prime}(s)\right)\right)}
$$

and

$$
\frac{\Delta}{K^{2}(s)} \Delta_{J_{1}}=\frac{J_{1}}{E^{2}(s)}+\frac{J_{1}\left(\frac{2 \lambda}{\pi^{2}}+\frac{\Delta^{2}}{K^{3}(s)} K^{\prime}(s)-\frac{J_{1}^{2}}{E^{3}(s)} E^{\prime}(s)\right)}{E^{2}(s)\left(\frac{J_{1}^{2} E^{\prime}(s)}{E^{3}(s)}-\frac{J_{2}^{2}}{(K(s)-E(s))^{3}}\left(K^{\prime}(s)-E^{\prime}(s)\right)\right)}
$$

After a very lengthy computation using various identities between elliptic functions and the relations

$$
w_{2}^{2}=\frac{4}{\pi^{2}} K^{2}(s)+w_{1}^{2}, \quad \kappa^{2}=\frac{4}{\pi^{2}} K^{2}(s) s+w_{1}^{2}
$$

\footnotetext{
${ }^{6}$ We base this intuition on the expectation of the structure of the heavy vertex operator to be of the form $e^{i J_{2} \phi_{2}}$ multiplied by terms with derivatives of $\phi_{2}$.
} 
we find the very simple result

$$
\frac{\partial \Delta\left(J_{1}, J_{2}\right)}{\partial J_{1}}=\frac{w_{1}}{\kappa}
$$

Incorporating this term in the integral (5.14), we thus get

$$
\int_{-\infty}^{\infty} \frac{d \tau}{\cosh ^{k} \kappa\left(\tau-\tau_{0}\right)} \int_{0}^{2 \pi} d \sigma e^{-w_{1} k\left(\tau-\tau_{0}\right)} \sin ^{k} \psi\left[\kappa^{2} \tanh ^{2} \kappa\left(\tau-\tau_{0}\right)+\left(\frac{\partial \vec{n}}{\partial \tau}\right)^{2}\right]
$$

Again we see, as in the previous case, that the dependence on $\tau_{0}$ can be undone in the $\tau$ integral and we recover the previous result of [38].

At this stage one might get the impression that the averaging over the moduli space together with the contribution of the heavy vertex operators is always trivial. However, as the next example shows this is not always the case.

\subsection{A two-magnon solution in finite volume}

In this section we will consider a finite volume two particle state with the particle momenta being of order $\mathcal{O}(1)$. Obtaining an exact finite-volume multi-magnon solution is a formidable endeavour (cf. [67, 68]), but we may obtain significant simplification when we neglect exponential corrections and concentrate on obtaining all power law finite size corrections.

This will be analogous to the situation of a single giant magnon. In that case, the exact finite volume solution [70] led just to the appearance of Lüscher exponential corrections [71]. If we would neglect them, we could just as well focus on the infinite volume solution.

The idea is thus to construct an approximate finite-volume two-particle solution by taking the exact infinite volume two particle solution and performing periodic identificiation with appropriate gluing. Since the large $\sigma$ fall-off of the multi-magnon solution is exponential, we can perform this procedure up to exponential accuracy so we should be able to recover all finite size power law corrections.

The two magnon solution has been constructed ${ }^{7}$ in [72] (see also [73] for generalizations to an arbitrary number of magnons). It is given explicitly by

$$
\begin{aligned}
X_{1}+i X_{2} & =e^{i t}+\frac{e^{i t}(R+i I)}{\sin \frac{p_{1}}{2} \sin \frac{p_{2}}{2}\left(1+\sinh u_{1} \sinh u_{2}\right)-\left(1-\cos \frac{p_{1}}{2} \cos \frac{p_{2}}{2}\right) \cosh u_{1} \cosh u_{2}} \\
X_{3} & =\frac{\left(\cos \frac{p_{1}}{2}-\cos \frac{p_{2}}{2}\right)\left(\sin \frac{p_{1}}{2} \cosh u_{2}-\sin \frac{p_{2}}{2} \cosh u_{1}\right)}{\sin \frac{p_{1}}{2} \sin \frac{p_{2}}{2}\left(1+\sinh u_{1} \sinh u_{2}\right)-\left(1-\cos \frac{p_{1}}{2} \cos \frac{p_{2}}{2}\right) \cosh u_{1} \cosh u_{2}}
\end{aligned}
$$

where

$$
\begin{aligned}
R & =\left(\cos \frac{p_{1}}{2}-\cos \frac{p_{2}}{2}\right)^{2} \cosh u_{1} \cosh u_{2} \\
I & =\left(\cos \frac{p_{1}}{2}-\cos \frac{p_{2}}{2}\right)\left(\sin \frac{p_{1}}{2} \sinh u_{1} \cosh u_{2}-\sin \frac{p_{2}}{2} \cosh u_{1} \sinh u_{2}\right)
\end{aligned}
$$

\footnotetext{
${ }^{7}$ Although in principle it arises by Pohlmeyer reduction from the well known two-soliton solution in sine-Gordon theory, obtaining the full explicit target space solution in $S^{3}$ is far from trivial.
} 
and $u_{1,2}$ are defined by

$$
u_{i}=\frac{s-t \cos \frac{p_{i}}{2}}{\sin \frac{p_{i}}{2}}
$$

For the case at hand, we will be interested in a state with vanishing total momentum, which is realized by taking $p_{1}=p$ and $p_{2}=2 \pi-p$. This solution can be readily compactified as $X_{3} \rightarrow 0$ and $X_{1}+i X_{2} \rightarrow-e^{i t}$ when $s \rightarrow \pm \infty$.

Due to the fact that for correlation functions we need to deal with Wick rotated solutions in Euclidean signature, it is convenient to analytically continue the momentum to $p=-i P$ and the size of the cylinder to purely imaginary values $L \rightarrow-i L$ (this amounts to taking $s=-i \sigma$ in addition to $t=-i \tau)$. At the very end of the computation we may take the final result and rotate back to physical values of the momentum and cylinder size.

The $u_{1,2}$ now take the form

$$
u_{1,2}=\frac{\sigma \mp \tau \cosh \frac{P}{2}}{\sinh \frac{P}{2}}
$$

In the present case we will compute the OPE coefficient with the Lagrangian density since firstly, we will be able to check the answer due to a general formula derived in [39], and secondly, since the corresponding vertex operator of the dilaton does not carry any Rcharges, the ingoing and outgoing classical states can coincide, and hence we will be dealing with the symmetric OPE's mentioned in section 4. This particular case of symmetric OPE's is of particular interest due to structural similarity with diagonal finite-volume form factors, which we will describe in section 6 .

The HHL formula with the dilaton vertex operator takes the form

$$
C_{\mathrm{HHL}}=\lim _{T \rightarrow \infty} \frac{1}{T} \int_{-\frac{T}{2}}^{\frac{T}{2}} d \tau_{0} \frac{3}{16} \int d \tau d \sigma \frac{1}{\cosh ^{4}\left(\tau-\tau_{0}\right)} \underbrace{\left[1+\partial X^{K} \bar{\partial} X^{K}\right]}_{F(\sigma, \tau)}
$$

where the factor $3 / 16$ is chosen in accordance with the normalization defined through

$$
C_{\mathrm{HHL}}=\frac{d}{d \frac{\sqrt{\lambda}}{\pi}} E
$$

which will be convenient for us.

Let us first change the integrations for $\tau_{0}$ and $\tau$ together with their domains:

$$
\begin{aligned}
C_{\mathrm{HHL}} & =\frac{3}{16} \lim _{T \rightarrow \infty} \frac{1}{T} \int_{-\frac{T}{2}}^{\frac{T}{2}} d \tau_{0} \int_{-\infty}^{\infty} d \tau \frac{1}{\cosh ^{4}\left(\tau-\tau_{0}\right)} \int d \sigma F(\sigma, \tau) \\
& =\frac{3}{16} \lim _{T \rightarrow \infty} \frac{1}{T} \int_{-\infty}^{\infty} d \tau \int_{-\frac{T}{2}}^{\frac{T}{2}} d \tau_{0} \frac{1}{\cosh ^{4}\left(\tau-\tau_{0}\right)} \int d \sigma F(\sigma, \tau)
\end{aligned}
$$

Now let us focus on the integral over $\tau_{0}$ assuming $T$ is large:

$$
\int_{-\frac{T}{2}}^{\frac{T}{2}} d \tau_{0} \frac{1}{\cosh ^{4}\left(\tau-\tau_{0}\right)}
$$


Its value will depend on the relative value of $\tau$ with respect to $T$. It is important to note that the integrand is exponentially suppressed for $\tau_{0}$ away from $\tau$. If $|\tau|$ is smaller than $T / 2$, then the integral essentially becomes independent of $\tau$ and is equal to a good accuracy to

$$
\int_{-\infty}^{\infty} \frac{d \tau^{\prime}}{\cosh ^{4} \tau^{\prime}}
$$

If, on the other hand, $|\tau|>T / 2$, the integral (5.31) just picks the exponential tail and is essentially zero. The above reasoning breaks down in a range in $\tau$ of width of order one around $\pm T / 2$. Since in any case we will be taking the limit $T \rightarrow \infty$ and multiplying by $1 / T$, the contribution of this transitional region will vanish in the limit. Hence in (5.30) we may make the substitution

$$
\int_{-\frac{T}{2}}^{\frac{T}{2}} d \tau_{0} \frac{1}{\cosh ^{4}\left(\tau-\tau_{0}\right)} \longrightarrow\left(\int_{-\infty}^{\infty} \frac{d \tau^{\prime}}{\cosh ^{4} \tau^{\prime}}\right) \cdot \theta\left(\frac{T^{2}}{4}-\tau^{2}\right)
$$

Thus (5.30) will simplify to

$$
\begin{aligned}
C_{\mathrm{HHL}} & =\frac{3}{16} \int_{-\infty}^{\infty} \frac{d \tau^{\prime}}{\cosh ^{4} \tau^{\prime}} \cdot \lim _{T \rightarrow \infty} \frac{1}{T} \int_{-\frac{T}{2}}^{\frac{T}{2}} d \tau \int d \sigma F(\sigma, \tau) \\
& =\frac{1}{4} \lim _{T \rightarrow \infty} \frac{1}{T} \int_{-\frac{T}{2}}^{\frac{T}{2}} d \tau \int d \sigma F(\sigma, \tau)
\end{aligned}
$$

For the application to the two magnon solution we still have to incorporate an additional factor of $(-i)$ due to our analytical continuation of the spatial worldsheet coordinate.

Let us note that in the case of the two magnon state, we have in principle also a relative time shift in the trajectories of the two individual particles (more precisely independent shifts in the $u_{1,2}$ variables). However the effect of this shift can be traded for a rigid worldsheet space and time translation. The worldsheet spatial translation clearly acts trivially in the HHL computation, especially as we are, in any case, integrating over the light vertex operator insertion point. For the case of multimagnon states with more than 2 particles, these relative shifts have to be taken into account and a more complicated structure emerges. We will consider this in detail in a forthcoming publication [76].

For the analytically continued two particle solution, the key expression entering formula (5.30) turns out to be

$$
F_{2 p}(\sigma, \tau)=\frac{32 \cosh ^{2} \frac{P}{2}\left(S_{1}-S_{2}\right)^{2}}{\left((3+\cosh P) C_{1} C_{2}+2 \sinh ^{2} \frac{P}{2}\left(1+S_{1} S_{2}\right)\right)^{2}}
$$

where we used the notation $C_{i}=\cosh u_{i}$ and $S_{i}=\sinh u_{i}$. When $\tau$ is positive and large, the 2-particle state becomes a superposition of two well separated magnons:

$$
F_{2 p}(\sigma, \tau) \rightarrow \frac{2}{\cosh ^{2}\left(u_{1}-\alpha\right)}+\frac{2}{\cosh ^{2}\left(u_{2}+\alpha\right)}
$$

with

$$
\sinh \alpha=\frac{\sinh ^{2} \frac{P}{2}}{2 \cosh \frac{P}{2}} ; \quad \alpha=\log \cosh \frac{P}{2}
$$


When $\tau$ is negative and large, we have on the other hand

$$
F_{2 p}(\sigma, \tau) \rightarrow \frac{2}{\cosh ^{2}\left(u_{1}+\alpha\right)}+\frac{2}{\cosh ^{2}\left(u_{2}-\alpha\right)}
$$

Each constituent particle moves with velocity $v=\cosh \frac{P}{2}$ and suffers from a (negative) time delay due to the 2-body interaction. Note that the rather unphysical details are due to our analytical continuation involving imaginary momenta and worldsheet spatial coordinate.

Suppose we now compactify the plane to a cylinder of size $L$. Since the $\tau$ integral in (5.30) will effectively pick out for us the period of motion $T_{0}$ through

$$
\lim _{T \rightarrow \infty} \frac{1}{T} \int_{-\frac{T}{2}}^{\frac{T}{2}} d \tau \quad \longrightarrow \quad \frac{1}{T_{0}} \int_{-\frac{T_{0}}{2}}^{\frac{T_{0}}{2}} d \tau
$$

let us now compute $T_{0}$ explicitly.

The trajectory of the first particle for $\tau>0$ is approximately given by $u_{1}+\alpha=0$ :

$$
\sigma=\tau \cosh \frac{P}{2}-\alpha \sinh \frac{P}{2}
$$

The period $T_{0}$ is determined by

$$
\frac{L}{2}=\left(\frac{T_{0}}{2}-2 \Delta T\right) \cosh \frac{P}{2}
$$

where $\Delta T=\alpha \tanh \frac{P}{2}$ (note that there are two interaction regions per period hence the factor 2 in $2 \Delta T$ - see figure 1 ). This gives

$$
T_{0}=\frac{L+4 \alpha \sinh \frac{P}{2}}{\cosh \frac{P}{2}}
$$

Finally let us determine the relation between the size of the cylinder $L$ in this setup and a similar quantity appearing in a Bethe Ansatz computation. This requires some care since, as already noted in [74], the giant magnon solution (and its multiparticle generalizations) is written in a different string gauge than the uniform light cone gauge employed in the Asymptotic Bethe Ansatz. Fortunately the relation is very simple. We get

$$
\left(L_{B A} \equiv\right) J=\frac{\sqrt{\lambda}}{2 \pi}(-i)\left(L-4 \sinh \frac{P}{2}\right)
$$

Note that at the classical level the 2-particle state exists for any value of $L$ and $P$. Bethe Ansatz quantization would only arise if we were to impose a WKB quantization condition. It is intriguing, however, that the purely classical HHL computation with the dilaton vertex operator will turn out to know about the precise form of the Bethe Ansatz quantization. 


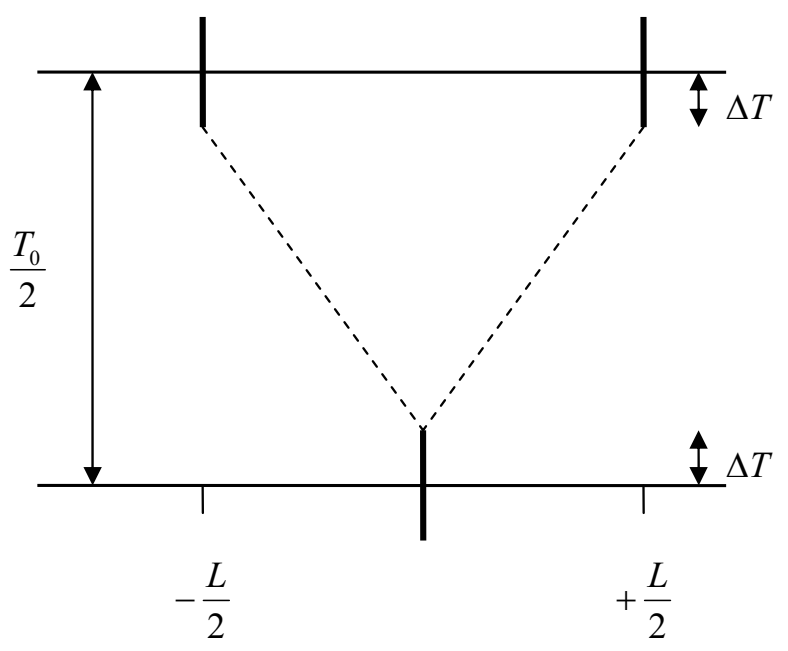

Figure 1. Schematic structure of the finite volume 2-particle state, arising from gluing together various parts of the infinite volume 2-particle classical solutions. $L$ is the circumference of the worldsheet cylinder, while $T_{0}$ is the period. The thick vertical lines represent the interaction regions, while the dashed lines represent (approximate up to exponential corrections) free propagation of the constituent particles.

The HHL computation for the 2-magnon state and the dilaton. Since the compactified finite volume solution is periodic with period $T_{0}$ given by $(5.42)$, we have to compute

$$
C_{\mathrm{HHL}}=\frac{-i}{4} \frac{1}{T_{0}} \int_{-\frac{T_{0}}{2}}^{\frac{T_{0}}{2}} d \tau \int d \sigma F_{2 p}(\sigma, \tau)
$$

It is convenient to rewrite $F_{2 p}(\sigma, \tau)$ in the following way, adding and subtracting the simple contributions of the individual constituent particles:

$$
F_{2 p}(\sigma, \tau)=F_{1 p}\left(u_{1} \pm \alpha\right)+F_{1 p}\left(u_{2} \mp \alpha\right)+\left[F_{2 p}(\sigma, \tau)-F_{1 p}\left(u_{1} \pm \alpha\right)-F_{1 p}\left(u_{2} \mp \alpha\right)\right]
$$

where the upper sign holds for $\tau>0$ and

$$
F_{1 p}(u)=\frac{2}{\cosh ^{2} u}
$$

The single particle integrals immediately yield

$$
\frac{-i}{4} \frac{1}{T_{0}} \int_{-\frac{T_{0}}{2}}^{\frac{T_{0}}{2}} d \tau \int d \sigma\left[F_{1 p}\left(u_{1} \pm \alpha\right)+F_{1 p}\left(u_{2} \mp \alpha\right)\right]=(-i) 2 \sinh \frac{P}{2}
$$

which is the infinite volume answer arising just from the dispersion relation of the two constituent magnons, which, in our analytical continuation take the form

$$
E=(-i) \frac{\sqrt{\lambda}}{\pi}\left(2 \times \sinh \frac{P}{2}\right)
$$

The expression in square brackets in (5.45) is concentrated only in the vicinity of the interaction point and is exponentially suppressed away from it. Hence in order to compute 
the integral, we can just add 2 contributions from the two interaction regions in each period and compute the contribution of a single interaction region extending the range of integration in $\sigma$ and $\tau$ to run from $-\infty$ to $\infty$ :

$$
\begin{array}{r}
\frac{-i}{4} \cdot 2 \times \frac{1}{T_{0}} \int_{-\frac{T_{0}}{2}}^{\frac{T_{0}}{2}} d \tau \int_{-\infty}^{\infty} d \sigma\left[F_{2 p}(\sigma, \tau)-F_{1 p}\left(u_{1} \pm \alpha\right)-F_{1 p}\left(u_{2} \mp \alpha\right)\right]= \\
\frac{-i}{4} \cdot 2 \times \frac{1}{T_{0}} \int_{-\infty}^{\infty} d \tau \int_{-\infty}^{\infty} d \sigma\left[F_{2 p}(\sigma, \tau)-F_{1 p}\left(u_{1} \pm \alpha\right)-F_{1 p}\left(u_{2} \mp \alpha\right)\right]
\end{array}
$$

We were unable to directly compute this integral analytically, however assuming that it can be expressed in terms of the phase shift $\alpha$ through some simple functions, we guessed the exact analytical answer from a numerical evaluation. Namely it is given by

$$
\begin{aligned}
& \frac{-i}{4} \cdot 2 \times \frac{1}{T_{0}} \int_{-\infty}^{\infty} d \tau \int_{-\infty}^{\infty} d \sigma\left[F_{2 p}(\sigma, \tau)-F_{1 p}\left(u_{1} \pm \alpha\right)-F_{1 p}\left(u_{2} \mp \alpha\right)\right]= \\
& \frac{-i}{4} \cdot 2 \times \frac{1}{T_{0}}[-16 \alpha \cosh \alpha(1+\tanh \alpha)]
\end{aligned}
$$

Since $\cosh \alpha(1+\tanh \alpha)=\cosh \frac{P}{2}$, the final answer for the HHL computation is

$$
C_{\mathrm{HHL}}=(-i)\left[2 \sinh P-\frac{8 \cosh ^{2} \frac{P}{2} \log \cosh \frac{P}{2}}{L+4 \sinh \frac{P}{2} \log \cosh \frac{P}{2}}\right]
$$

The denominator in the above expression comes from averaging over the period (the $1 / T_{0}$ term). Performing a large $L$ expansion, this expression already includes a precise expression for all power law finite size corrections.

Bethe ansatz prediction for $\boldsymbol{C}_{\mathbf{H H L}}$. Let us now compare the result (5.51) with the Bethe ansatz prediction taking into account the relation (5.29).

The energy of the two particle state is given by

$$
E=E\left(p_{1}\right)+E\left(p_{2}\right)
$$

where we neglect any wrapping effects. The derivative w.r.t. $\sqrt{\lambda} / \pi$ will get two contributions, one coming from the explicit dependence of the dispersion relation on the coupling, and another, characteristic of finite size, coming from the change in the momenta implied by the modification of the Bethe ansatz quantization

$$
e^{i p L_{B A}+i \delta(p,-p)}=1
$$

through the dependence of the S-matrix (here parametrized in terms of the strong coupling classical phase shift $\delta(p,-p))$ on the coupling. The result of this computation is

$$
E^{\prime}\left(p_{1}\right)+E^{\prime}\left(p_{2}\right)-\frac{\delta^{\prime} \frac{\partial E}{\partial p_{1}}}{L_{B A}+\delta_{1}-\delta_{2}}+\frac{\delta^{\prime} \frac{\partial E}{\partial p_{2}}}{L_{B A}+\delta_{1}-\delta_{2}}
$$

where the prime denotes derivative w.r.t. $\sqrt{\lambda} / \pi$ and $\delta_{i}=\partial \delta / \partial p_{i}$. The giant magnon phase shift is given by [74]

$$
\delta_{\mathrm{HM}}\left(p_{1}, p_{2}\right)=-\frac{\sqrt{\lambda}}{\pi}\left(\cos \frac{p_{1}}{2}-\cos \frac{p_{2}}{2}\right) \log \frac{\sin ^{2} \frac{p_{1}-p_{2}}{4}}{\sin ^{2} \frac{p_{1}+p_{2}}{4}}
$$


As explained in [74], the above formula applies when both momenta are positive. When we want to make one of the momenta negative we should use the substitution $p \rightarrow 2 \pi-p$. Thus the relevant phase shift becomes

$$
\delta(p,-p) \equiv \delta_{\mathrm{HM}}(p, 2 \pi-p)=-4 \frac{\sqrt{\lambda}}{\pi} \cos \frac{p}{2} \log \cos \frac{p}{2}
$$

In the following we will use imaginary momenta and radii

$$
p=-i P \quad L_{B A}=-i \tilde{L}
$$

Then we have

$$
\delta^{\prime}=-4 \cosh \frac{P}{2} \log \cosh \frac{P}{2}
$$

and

$$
\delta_{1}=-\delta_{2}=-i \frac{\sqrt{\lambda}}{\pi} \sinh \frac{P}{2}\left(1+\log \cosh \frac{P}{2}\right)
$$

The 2-particle prediction for $C_{\mathrm{HHL}}$ in our normalization thus takes the form

$$
(-i)\left[2 \sinh \frac{P}{2}-\frac{4 \cosh ^{2} \frac{P}{2} \log \cosh \frac{P}{2}}{\frac{\pi}{\sqrt{\lambda}} \tilde{L}+2 \sinh \frac{P}{2}+2 \sinh \frac{P}{2} \log \cosh \frac{P}{2}}\right]
$$

Note that the BA length is identified with the $J$ charge (any differences due to different sectors can be neglected in the strong coupling limit).

Let us now go back to the result of our classical HHL computation (5.51). Using the relations (5.57) and (5.43) we obtain

$$
\frac{\pi}{\sqrt{\lambda}} \tilde{L}=\frac{L}{2}-2 \sinh \frac{P}{2}
$$

Under this identification, the Bethe Ansatz prediction (5.60) and our modified HHL computation (5.51) exactly coincide. Let us emphasize, that the averaging over $\tau_{0}$ was absolutely crucial for obtaining the correct result, and the infinite set of power law finite size corrections was encoded in the period appearing in the denominator coming from that averaging. We will comment on the structural properties of the above solution in the following section.

\section{Symmetric OPE coefficients and diagonal formfactors}

We have seen in the previous sections that diagonal matrix elements of operators can be calculated by averaging the operator over the moduli space of the classical solutions. We argued that the classical limit of the OPE coefficient, $C_{\mathrm{HHL}}$, can be calculated by this procedure and performed the average of the dilaton vertex operator for the 2-magnon case explicitly. Integration over the moduli space resulted in the volume dependence of the average and the result incorporated all polynomial finite size corrections. This average in the quantum theory should correspond to the diagonal matrix element of the operator between multiparticle states. As the states are in finite volume the finite volume diagonal matrix elements are needed $[77,78]$. In the following we summarize what is known about 
these matrix elements ${ }^{8}$ and point out the structural similarities with the OPE coefficients. We restrict the investigations for one and two particle states as we calculated the structure constants up to this level.

We denote the finite volume one particle state with momentum $p$ as $|p\rangle_{L}$. The diagonal matrix element of a local operator $\mathcal{O}$ between this finite volume state up to exponentially small corrections is given by

$$
{ }_{L}\langle p|\mathcal{O}| p\rangle_{L}=\frac{1}{\rho_{1}(p)}\left(F_{1}^{\mathcal{O}}(p)+\rho_{1}(p) F_{0}^{\mathcal{O}}\right)
$$

where $F_{0}^{\mathcal{O}}$ is the infinite volume VEV, while $F_{1}^{\mathcal{O}}$ is the infinite volume diagonal one particle form factor. The volume dependence comes only from the density of one particle states:

$$
\rho_{1}(p)=L
$$

which is related to the normalization of the finite volume state.

A similar formula for a finite volume two particle state, labeled by $\left|p_{1}, p_{2}\right\rangle_{L}$, is given by

$$
{ }_{L}\left\langle p_{2}, p_{1}|\mathcal{O}| p_{1}, p_{2}\right\rangle_{L}=\frac{F_{2}^{\mathcal{O}}\left(p_{1}, p_{2}\right)+\rho_{1}\left(p_{1}\right) F_{1}^{\mathcal{O}}\left(p_{2}\right)+\rho_{1}\left(p_{2}\right) F_{1}^{\mathcal{O}}\left(p_{1}\right)}{\rho_{2}\left(p_{1}, p_{2}\right)}
$$

where we assumed that the VEV is vanishing and denoted the infinite volume two particle diagonal form factor by $F_{2}^{\mathcal{O}}$. The density of two-particle states is defined from the asymptotic Bethe Ansatz equation as

$$
\rho_{2}\left(p_{1}, p_{2}\right)=\operatorname{det}\left[\begin{array}{cc}
L+\phi_{12} & -\phi_{21} \\
-\phi_{12} & L+\phi_{21}
\end{array}\right]=L\left(L+\phi_{21}+\phi_{12}\right)
$$

where $\phi_{k l}$ is the logarithmic derivative of the S-matrix:

$$
\phi_{k l}=-i \frac{\partial \log S\left(p_{k}, p_{l}\right)}{\partial p_{k}}=i \frac{\partial \log S\left(p_{l}, p_{k}\right)}{\partial p_{k}}
$$

All the volume dependence of the matrix element come from the various densities, which are related to the finite volume normalization of the states.

Let us emphasize that the expression for the finite volume diagonal matrix elements eq. (6.1) and (6.3) in terms of the infinite volume form factors are quite general, valid for any local operator $\mathcal{O}$. In particular, the volume dependence always comes from the various density of states in an operator independent way, which is controlled by the asymptotic Bethe Ansatz equations. The difficult part is the calculation of the infinite volume form factors from first principles. This programme should include the determination and classification of the solutions of the form factor equations listed in $[67,68]$, which is very technical and demanding, although a free field representation along the lines of [75] could help in this problem.

The diagonal form factors, however, are much simpler than the general ones and we might have a hope to determine them exactly. Additionally, if the operator is related

\footnotetext{
${ }^{8} \mathrm{~A}$ detailed and self-contained exposition can be found in appendix A.
} 
to some conserved charge then its diagonal form factors are easy to calculate, which we demonstrate now.

Let us start with the form factor of the density of a conserved charge

$$
Q=\int_{0}^{L} \mathcal{O}(x, t) d x
$$

with the following explicitly known finite volume diagonal matrix element

$$
{ }_{L}\left\langle p_{1}, \ldots, p_{n}|\mathcal{O}| p_{n}, \ldots p_{1}\right\rangle_{L}=\frac{1}{L} \sum_{i} o\left(p_{i}\right)
$$

Using the parametrization of the finite volume matrix element in terms of the infinite volume form factors in eq. (6.1) and (6.3) we can systematically extract:

$$
F_{0}^{\mathcal{O}}=0 ; \quad F_{1}^{\mathcal{O}}=o_{1} ; \quad F_{2}^{\mathcal{O}}=\left(o_{1}+o_{2}\right)\left(\phi_{12}+\phi_{21}\right)
$$

where $o_{i}=o\left(p_{i}\right)$.

A similar case is when the operator is related to the derivative of a conserved charge w.r.t. some parameter. The dilaton is such an operator and we determine the form factors of its density:

$$
{ }_{L}\left\langle p_{1}, \ldots, p_{n}|\mathcal{D}| p_{n}, \ldots p_{1}\right\rangle_{L}=\frac{1}{L} \frac{d}{d g} \sum_{i} E\left(p_{i}(g), g\right)=\frac{1}{L} \sum_{i}\left(\frac{\partial E}{\partial g}+\frac{\partial E}{\partial p_{i}} \frac{d p_{i}}{d g}\right)
$$

By exploiting the parametrizations in eq. (6.1) and (6.3) we obtained the following form factors

$$
\begin{aligned}
& F_{0}^{\mathcal{D}}=0 ; \quad F_{1}^{\mathcal{D}}=\frac{\partial E}{\partial g} \\
& F_{2}^{\mathcal{D}}=\left(\frac{\partial E_{1}}{\partial g}+\frac{\partial E_{2}}{\partial g}\right)\left(\phi_{12}+\phi_{21}\right)+\left(\psi_{12} \frac{\partial E_{2}}{\partial p_{2}}+\psi_{21} \frac{\partial E_{1}}{\partial p_{1}}\right)
\end{aligned}
$$

where

$$
-i \partial_{g} \log S\left(p_{i}, p_{j}\right)=\psi_{i j}=-\psi_{j i}
$$

Using these finite volume form factors and the general expression (6.3) we can express the diagonal two particle matrix element of the vertex operator of the dilaton. The result is valid for any coupling and agrees in the strong coupling limit with the direct calculation of the structure constant $C_{\mathrm{HHL}}$.

Based on the agreement between the dilaton diagonal matrix element and the structure constant in the strong coupling limit we conjecture that the $C_{\mathrm{HHL}}$ structure constants correspond to diagonal finite volume form factors of the vertex operator of the light operator. Thus, for any light operator with vertex operator $\mathcal{O}$ and heavy operator correponding to a finite volume two magnon state with momentum $p_{1}$ and $p_{2}$ (satisfying the asymptotic BA equation) the all loop result should take the form

$$
C_{\mathrm{HHO}}=\frac{F_{2}^{\mathcal{O}}\left(p_{1}, p_{2}\right)+L F_{1}^{\mathcal{O}}\left(p_{2}\right)+L F_{1}^{\mathcal{O}}\left(p_{1}\right)}{L+\phi_{12}+\phi_{21}}
$$

where $F_{1}^{\mathcal{O}}, F_{2}^{\mathcal{O}}$ are the infinite volume diagonal one an two particle form factors. This expression contains all polynomial corrections in the inverse of the volume, but not the exponentially small wrapping contributions. 


\section{$7 \quad$ A conjecture}

Based on our explicit calculation for the dilaton diagonal matrix elements sandwiched between two particle states we conjecture that the $C_{\mathrm{HHL}}$ structure constants correspond to the diagonal finite volume form factors of the vertex operator of the light operator. Let the heavy operator correspond to a multiparticle state in a finite - but large - volume $L$, such that exponentially small vacuum polarization effects can be neglected. The energy in this approximation comes from the dispersion relation

$$
H\left|p_{1}, \ldots, p_{n}\right\rangle_{L}=\sum_{k=1}^{n} E\left(p_{k}\right)\left|p_{1}, \ldots, p_{n}\right\rangle_{L}
$$

and the momenta are quantized by the asymptotic Bethe ansatz equations:

$$
\Phi_{k}=p_{k} L-i \sum_{j: j \neq k} \log S\left(p_{k}, p_{j}\right)=2 \pi I_{k}
$$

where we assumed that the particles scatter diagonally. We conjecture that for a light operator with vertex operator $\mathcal{O}$ the structure constant is related to the final volume diagonal matrix element as

$$
\begin{aligned}
C_{\mathrm{HHL}} & ={ }_{L}\left\langle p_{1}, \ldots, p_{n}|\mathcal{O}| p_{n}, \ldots p_{1}\right\rangle_{L} \\
& =\frac{1}{\rho\{1, \ldots, n\}} \sum_{A \subseteq\{1, \ldots, n\}} \rho\{A\} F_{|\bar{A}|}^{\mathcal{O}}\{\bar{A}\} \\
& =\frac{F_{n}^{\mathcal{O}}+\sum_{i} \rho\{i\} F_{n-1}^{\mathcal{O}}\{1, \ldots, \hat{i}, \ldots n\}+\sum_{i, j} \rho\{i, j\} F_{n-2}^{\mathcal{O}}\{1, \ldots, \hat{i}, \ldots, \hat{j}, \ldots, n\}+\ldots}{\rho\{1, \ldots, n\}}
\end{aligned}
$$

where $\bar{A}$ is the complement of $A$ i.e. $\bar{A}=\{1, \ldots, n\} \backslash A$. The volume dependence comes only from the asymptotic BA equation via the normalization of the finite volume states through the following subdeterminants:

$$
\rho\left\{i_{1}, \ldots, i_{m}\right\}=\operatorname{det}\left[\frac{\partial \Phi_{i_{k}}}{\partial p_{i_{j}}}\right]=\operatorname{det}\left[\frac{\partial}{\partial p_{i_{j}}}\left\{p_{i_{k}} L-i \sum_{l: l \neq k}^{m} \log S\left(p_{i_{k}}, p_{i_{l}}\right)\right\}\right]
$$

The diagonal form factors are defined as

$$
F_{k}^{\mathcal{O}}\left(i_{1}, \ldots, i_{k}\right)=\lim _{\epsilon \rightarrow 0}\left\langle 0|\mathcal{O}(0,0)| \bar{p}_{i_{k}}, \ldots, \bar{p}_{i_{1}}, p_{i_{1}}+\epsilon, \ldots, p_{i_{k}}+\epsilon\right\rangle
$$

and can (in principle) be calculated from an infinite volume axiomatic formulations [67, 68].

\section{Conclusions}

In this paper we focused on the determination of the OPE coefficients for heavy-heavy-light operators in the $\mathcal{N}=4$ Super-Yang-Mills theory. Our goal was to develop a framework which is valid not only in the weak coupling (spin-chain) or strong coupling (classical) limiting cases but allow for an interpolation between them. In so doing we investigated 
how quantum expectation values on energy eigenstates show up in the classical limit. We found that, in contrast to the coherent state approach when the time dependence of a single classical solution is described, here we have to integrate the observable over the moduli space of classical solutions with the given energy and other quantum numbers. We used this modified prescription to calculate the HHL coefficients in the strong coupling limit in three particular cases: for the BMN vacuum correlator, for folded strings on $S^{5}$ and for classical solutions with two particles. Our novel prescription reduces to the traditional one $[38,39]$ for simple solutions but differs from it in general. In particular, implementing the prescription for two-particle states with the dilaton/lagrangian density we obtained a dependence on the volume (size of the cylinder), which is exactly of the structure expected for finite volume diagonal formfactors. Moreover, this volume dependence arose in a way manifestly independent of the light operator. Based on this observation we conjectured that for heavy states corresponding to multiparticle states in large volume the $C_{\mathrm{HHL}}$ structure constants are related to the diagonal finite volume form factors of the vertex operator of the light operator. Our conjecture is applicable for asymptotically large volumes and incorporates all polynomial finite size corrections in the inverse of the volume, but neglects the exponentially small wrapping effects.

Our investigation focused on a particular two particle state and used a novel prescription to calculate the classical limit of the structure constant. It is desirable, however, to extend the analysis for generic multiparticle states and compare the result with the generic form of the finite volume diagonal multiparticle form factors. We have already initiated research into this direction [76].

The structure constants in the weak coupling limit can be calculated from a spin chain description [22]-[29]. As our results are conjectured to be valid for any coupling it is very challenging to test them against these results.

Our conjecture relates the HHL structure constants to diagonal form factors. As the theory of form factors was already initiated in $[67,68]$ for the AdS/CFT setting it would be extremely interesting to determine the generic multiparticle form factors of the dilaton by solving the form factor equations and to compare their diagonal limits to our expressions. We showed in the WKB approximation that the quantum expectation value in an energy eigenstate is related to the time average for the corresponding classical solution. It would be interesting to extend this argumentation explicitly for the finite volume multiparticle configurations of field theories.

Finally, it would be important to investigate whether such phenomena as averaging over the moduli space would resurface in the case of three Heavy operators. The HHL examples with spinning strings considered in the present paper seem to suggest that such effects would probably cancel out but it would be interesting to verify this explicitly within the setting of [33].

\section{Acknowledgments}

We would like to thank Konstantin Zarembo for interesting discussions during the research program Integrability and Gauge/String duality at the Institute for Advanced Studies in 
Jerusalem. We thank Shota Komatsu for important comments. R.J. and A.W. were supported by NCN grant 2012/06/A/ST2/00396. Z.B. was supported by OTKA 81461 and by a Lendület Grant. We acknowledge support from a Hungarian Academy of Sciences/Polish Academy of Sciences joint research project.

\section{A Finite volume diagonal form factors}

In this appendix we review the theory of finite volume diagonal form factors with one and two particles. The general theory and its relation to the classical solutions will be explained in a forthcoming publication. We rely on [77], but see also [78]. In [77] the authors analyzed the polynomial type finite size dependence of diagonal form factors and observed that the volume dependence comes only from the normalization of states.

\section{A.1 One particle diagonal matrix elements}

We start to define infinite volume 1-particle states and form factors and then express the finite volume quantities in terms of the infinite volume ones and the asymptotic Bethe equations.

An infinite volume one particle state can be labeled by its momentum, $p$. It is the eigenstate of the energy and momentum:

$$
P|p\rangle=p|p\rangle ; \quad H|p\rangle=E(p)|p\rangle
$$

States with different momenta are ortogonal, and we choose the following normalization ${ }^{9}$

$$
\left\langle p \mid p^{\prime}\right\rangle=2 \pi \delta\left(p-p^{\prime}\right)
$$

The diagonal matrix element of a local operator, $\mathcal{O}$, is denoted as:

$$
\langle p|\mathcal{O}| p\rangle \equiv\langle p|\mathcal{O}(x, t)| p\rangle=\langle p|\mathcal{O}(0,0)| p\rangle
$$

As the Hamiltonian generates time, while the momentum space translation, the diagonal matrix element does not depend on the position of the insertion of the operator, it is a function of the momentum of the external state only. The crossing equation of the form factors tells us how we can move a particle from the final state into the initial one. In doing so disconnected terms appear, which for the 1-particle case read

$$
\left\langle p|\mathcal{O}| p^{\prime}\right\rangle=\left\langle 0|\mathcal{O}| \bar{p}, p^{\prime}\right\rangle+\left\langle p \mid p^{\prime}\right\rangle\langle\mathcal{O}\rangle
$$

where we denoted by $\bar{p}$ the crossed momentum. Clearly if the field has a VEV,$F_{0}^{\mathcal{O}} \equiv$ $\langle\mathcal{O}\rangle \neq 0$, the diagonal matrix element is not well-defined with the normalization (A.2). Nevertheless, we can introduce the finite part:

$$
F_{1}^{\mathcal{O}}(p)=\lim _{\epsilon \rightarrow 0}\langle 0|\mathcal{O}| \bar{p}, p+\epsilon\rangle
$$

\footnotetext{
${ }^{9}$ In relativistic theories a more natural normalization would be $\left\langle p \mid p^{\prime}\right\rangle=2 \pi E(p) \delta\left(p-p^{\prime}\right)$.
} 
so that formally we can write

$$
\langle p|\mathcal{O}| p\rangle=F_{1}^{\mathcal{O}}(p)+\langle p \mid p\rangle F_{0}^{\mathcal{O}}
$$

In the following we analyze the one particle state in a finite, but large volume, such that the exponentially supressed vacuum polarization effects can be safely neglected. In this approximation the dispersion relation is not changed, but the momentum is quantized by the periodicity of the wavefunction

$$
\Phi_{1}=p L=2 \pi n
$$

The finite volume states can be labeled by the quantization number $n$ and they are also eigenvectors of the energy and momentum:

$$
|p\rangle_{L}:=|n\rangle ; \quad P|p\rangle_{L}=p|p\rangle_{L} ; \quad H|p\rangle_{L}=E(p)|p\rangle_{L}
$$

As the spectrum is discrete a natural normalization is different from the one we used in infinite volume:

$$
\left\langle n \mid n^{\prime}\right\rangle=\delta_{n, n^{\prime}}
$$

In the large volume limit the spectrum is very dense and we can relate the two normalizations via (A.7)

$$
\sum_{n}|n\rangle\left\langle n\left|\approx \int \frac{d p}{2 \pi} L\right| p\right\rangle_{L} L\left\langle p\left|=\int \frac{d p}{2 \pi}\right| p\right\rangle\langle p|
$$

as

$$
|p\rangle_{L}=\frac{1}{\sqrt{\rho_{1}}}|p\rangle ; \quad \rho_{1}(p)=L
$$

In very large volume the finite volume diagonal matrix element differs from the infinite volume one only by the normalization of states:

$$
{ }_{L}\langle p|\mathcal{O}| p\rangle_{L}=\frac{1}{\rho_{1}(p)}\left(F_{1}^{\mathcal{O}}(p)+\rho_{1}(p) F_{0}^{\mathcal{O}}\right)
$$

so that all the volume dependence comes through the normalizations [77]. For the later applications we assume that operators do not have any VEVs.

\section{A.2 Two particle diagonal matrix elements}

In infinite volume the initial and final states are special: as well separated particles do not interact multiparticle states behave like free states. These states in the two particle case are labeled by their momenta $p_{1}, p_{2}$. In the initial state the faster particle is on the left so we denote this state by $\left|p_{1}, p_{2}\right\rangle$ assuming that $p_{1}>p_{2}$. The final state, when the faster is on the right, is denoted by $\left|p_{2}, p_{1}\right\rangle$. They are connected by the two particle S-matrix

$$
\left|p_{1}, p_{2}\right\rangle=S\left(p_{1}, p_{2}\right)\left|p_{2}, p_{1}\right\rangle
$$

We can formally extend the fundamental domain of the scattering matrix from $p_{1}>p_{2}$ by maintaining the relation (A.13):

$$
S\left(p_{1}, p_{2}\right) S\left(p_{2}, p_{1}\right)=1
$$


Both the initial and final two particle states are energy eigenstates, which are normalized as

$$
\left\langle p_{2}, p_{1} \mid p_{1}^{\prime}, p_{2}^{\prime}\right\rangle=(2 \pi)^{2} \delta\left(p_{1}-p_{1}^{\prime}\right) \delta\left(p_{2}-p_{2}^{\prime}\right)
$$

Here we assumed that $p_{1}>p_{2}$ and $p_{1}^{\prime}>p_{2}^{\prime}$.

The diagonal matrix element between initial and final states does not depend on the insertion point:

$$
\left\langle p_{2}, p_{1}|\mathcal{O}| p_{1}, p_{2}\right\rangle \equiv\left\langle p_{2}, p_{1}|\mathcal{O}(0,0)| p_{1}, p_{2}\right\rangle
$$

The crossing relation with the disconnected pieces reads as

$$
\begin{aligned}
\left\langle p_{2}, p_{1}|\mathcal{O}| p_{1}^{\prime}, p_{2}^{\prime}\right\rangle= & \left\langle p_{2}|\mathcal{O}| \bar{p}_{1}, p_{1}^{\prime}, p_{2}^{\prime}\right\rangle \\
& +\left\langle p_{1} \mid p_{1}^{\prime}\right\rangle\left\langle p_{2}|\mathcal{O}| p_{2}^{\prime}\right\rangle+S\left(p_{1}^{\prime}, p_{2}^{\prime}\right)\left\langle p_{1} \mid p_{2}^{\prime}\right\rangle\left\langle p_{2}|\mathcal{O}| p_{1}^{\prime}\right\rangle
\end{aligned}
$$

Crossing the other term and keeping only those which survive in the diagonal limit we obtain:

$$
\begin{aligned}
\left\langle p_{2}, p_{1}|\mathcal{O}| p_{1}^{\prime}, p_{2}^{\prime}\right\rangle= & \left\langle 0|\mathcal{O}| \bar{p}_{2}, \bar{p}_{1}, p_{1}^{\prime}, p_{2}^{\prime}\right\rangle \\
& +\left\langle p_{1} \mid p_{1}^{\prime}\right\rangle\left\langle 0|\mathcal{O}| \bar{p}_{2}, p_{2}^{\prime}\right\rangle+\left\langle p_{2} \mid p_{2}^{\prime}\right\rangle\left\langle 0|\mathcal{O}| \bar{p}_{1}, p_{1}^{\prime}\right\rangle+\ldots
\end{aligned}
$$

In taking the diagonal limit we have to be careful. Firstly, the disconnected terms will involve delta functions. Secondly, the first term depends on the way in which we take the limit. Choosing the symmetric evaluation:

$$
F_{2}^{\mathcal{O}}\left(p_{1}, p_{2}\right)=\lim _{\epsilon \rightarrow 0}\left\langle 0|\mathcal{O}| \bar{p}_{2}, \bar{p}_{1}, p_{1}+\epsilon, p_{2}+\epsilon\right\rangle
$$

leads to the following formal expression for the diagonal two particle form factor:

$$
\left\langle p_{2}, p_{1}|\mathcal{O}| p_{1}, p_{2}\right\rangle=F_{2}^{\mathcal{O}}\left(p_{1}, p_{2}\right)+\left\langle p_{1} \mid p_{1}\right\rangle F_{1}^{\mathcal{O}}\left(p_{2}\right)+\left\langle p_{2} \mid p_{2}\right\rangle F_{1}^{\mathcal{O}}\left(p_{1}\right)
$$

We now give meaning to this formula by putting the system in a finite volume.

In a finite but large volume, the momenta are quantized by the Bethe-Yang equations:

$$
\begin{aligned}
& \Phi_{1} \equiv p_{1} L-i \log S\left(p_{1}, p_{2}\right)=2 \pi n_{1} \\
& \Phi_{2} \equiv p_{2} L-i \log S\left(p_{2}, p_{1}\right)=2 \pi n_{2}
\end{aligned}
$$

The finite volume two particle state is a scattering state, symmetric in the momenta, which is labeled by

$$
\left|p_{1}, p_{2}\right\rangle_{L}=\left|n_{1}, n_{2}\right\rangle ; \quad n_{1}>n_{2}
$$

and normalized as

$$
\left\langle n_{2}, n_{1} \mid n_{1}^{\prime}, n_{2}^{\prime}\right\rangle=\delta_{n_{1} n_{1}^{\prime}} \delta_{n_{2}, n_{2}^{\prime}}
$$

The finite volume state is related to the infinite volume one via

$$
\begin{aligned}
\sum_{n_{1}>n_{2}}\left|n_{1}, n_{2}\right\rangle\left\langle n_{2}, n_{1}\right| & \approx \int_{-\infty}^{\infty} \frac{d p_{2}}{2 \pi} \int_{p_{2}}^{\infty} \frac{d p_{1}}{2 \pi} \operatorname{det}\left[\frac{\partial \Phi_{j}}{\partial p_{i}}\right]\left|p_{1}, p_{2}\right\rangle_{L}{ }_{L}\left\langle p_{2}, p_{1}\right| \\
& =\int_{-\infty}^{\infty} \frac{d p_{2}}{2 \pi} \int_{p_{2}}^{\infty} \frac{d p_{1}}{2 \pi}\left|p_{1}, p_{2}\right\rangle\left\langle p_{2}, p_{1}\right|
\end{aligned}
$$


$\operatorname{as}^{10}$

$$
\left|p_{1}, p_{2}\right\rangle_{L}=\frac{1}{\sqrt{S\left(p_{1}, p_{2}\right) \rho_{2}\left(p_{1}, p_{2}\right)}}\left|p_{1}, p_{2}\right\rangle
$$

Here

$$
\rho_{2}\left(p_{1}, p_{2}\right)=\operatorname{det}\left[\begin{array}{cc}
L+\phi_{12} & -\phi_{21} \\
-\phi_{12} & L+\phi_{21}
\end{array}\right]=L\left(L+\phi_{21}+\phi_{12}\right)
$$

and

$$
\phi_{k l}=-i \frac{\partial \log S\left(p_{k}, p_{l}\right)}{\partial p_{k}}=i \frac{\partial \log S\left(p_{l}, p_{k}\right)}{\partial p_{k}}
$$

The finite volume diagonal matrix element can be written in terms of the infinite volume form factors as

$$
{ }_{L}\left\langle p_{2}, p_{1}|\mathcal{O}| p_{1}, p_{2}\right\rangle_{L}=\frac{F_{2}^{\mathcal{O}}\left(p_{1}, p_{2}\right)+\rho_{1}\left(p_{1}\right) F_{1}^{\mathcal{O}}\left(p_{2}\right)+\rho_{1}\left(p_{2}\right) F_{1}^{\mathcal{O}}\left(p_{1}\right)}{\rho_{2}\left(p_{1}, p_{2}\right)}
$$

where the volume dependence comes only through the densities $\rho_{i}$.

\section{A.3 Form factors of special operators}

The calculation of the infinite volume form factors is a very technical and complicated problem. One should start with the form factor equations listed in $[67,68]$ and find the relevant solution for a given operator recursively in the particle number. If, however, the operator is related to some conserved charge, its diagonal form factor is very easy to calculate and in the following we focus on them.

As a starting point we consider the density of a conserved charge:

$$
Q=\int_{0}^{L} \mathcal{O}(x, t) d x
$$

with the following explicitly known finite volume diagonal matrix element ${ }^{11}$

$$
{ }_{L}\left\langle p_{1}, \ldots, p_{n}|\mathcal{O}| p_{n}, \ldots p_{1}\right\rangle_{L}=\frac{1}{L} \sum_{i} o\left(p_{i}\right)
$$

We can thus systematically extract the infinite volume form factors as follows:

$$
F_{0}^{\mathcal{O}}=0 ; \quad F_{1}^{\mathcal{O}}=o_{1} ; \quad F_{2}^{\mathcal{O}}=\left(o_{1}+o_{2}\right)\left(\phi_{12}+\phi_{21}\right)
$$

where $o_{i}=o\left(p_{i}\right)$.

We analyze now an operator which is related to the derivative of a conserved charge w.r.t. some parameter, which we denote by $g$. We assume that the BY equations will depend on this parameter and it affects the conserved charge via the momenta: ${ }^{12}$

$$
{ }_{L}\left\langle p_{1}, \ldots, p_{n}|\mathcal{O}| p_{n}, \ldots p_{1}\right\rangle_{L}=\frac{1}{L} \frac{d}{d g} \sum_{i} o\left(p_{i}(g)\right)=\frac{1}{L} \sum_{i} \frac{d o\left(p_{i}\right)}{d p_{i}} \frac{d p_{i}}{d g}
$$

\footnotetext{
${ }^{10}$ Similar normalization has been suggested in [26].

${ }^{11}$ Again valid up to exponential finite size corrections.

${ }^{12}$ If the operator depends on $g$ explicitly the calculation of the $\partial o / \partial g$ part can be reduced to the previous case.
} 
The derivative of the momenta can be expressed from the derivative of the BY equations w.r.t. $g$.

$$
0=\frac{d \Phi_{i}}{d g}=\frac{\partial \Phi_{i}}{\partial g}+\frac{d p_{j}}{d g} \partial_{j} \Phi_{i}
$$

We will assume that only the scattering matrix depends on the parameter $g$ and denote its derivatives by

$$
-i \partial_{g} \log S\left(p_{i}, p_{j}\right)=\psi_{i j}=-\psi_{j i}
$$

The diagonal form factors can be extracted as:

$$
F_{0}^{\mathcal{O}}=F_{1}^{\mathcal{O}}=0 ; \quad F_{2}^{\mathcal{O}}=\psi_{12} o_{2}^{\prime}+\psi_{21} o_{1}^{\prime}
$$

In particular for the dilaton we have to combine the two descriptions as it is related to derivative of the energy w.r.t. the coupling constant:

$$
{ }_{L}\left\langle p_{1}, \ldots, p_{n}|L \mathcal{D}| p_{n}, \ldots p_{1}\right\rangle_{L}=\frac{d}{d g} \sum_{i} E\left(p_{i}(g), g\right)=\sum_{i}\left(\frac{\partial E}{\partial g}+\frac{\partial E}{\partial p_{i}} \frac{d p_{i}}{d g}\right)
$$

Extracting the infinite volume form factors one obtains

$$
\begin{aligned}
& F_{0}^{\mathcal{D}}=0 ; \quad F_{1}^{\mathcal{D}}=\frac{\partial E}{\partial g} \\
& F_{2}^{\mathcal{D}}=\left(\frac{\partial E_{1}}{\partial g}+\frac{\partial E_{2}}{\partial g}\right)\left(\phi_{12}+\phi_{21}\right)+\left(\psi_{12} \frac{\partial E_{2}}{\partial p_{2}}+\psi_{21} \frac{\partial E_{1}}{\partial p_{1}}\right)
\end{aligned}
$$

Open Access. This article is distributed under the terms of the Creative Commons Attribution License (CC-BY 4.0), which permits any use, distribution and reproduction in any medium, provided the original author(s) and source are credited.

\section{References}

[1] J.M. Maldacena, The large-N limit of superconformal field theories and supergravity, Adv. Theor. Math. Phys. 2 (1998) 231 [Int. J. Theor. Phys. 38 (1999) 1113] [hep-th/9711200] [INSPIRE].

[2] S.S. Gubser, I.R. Klebanov and A.M. Polyakov, Gauge theory correlators from noncritical string theory, Phys. Lett. B 428 (1998) 105 [hep-th/9802109] [INSPIRE].

[3] E. Witten, Anti-de Sitter space and holography, Adv. Theor. Math. Phys. 2 (1998) 253 [hep-th/9802150] [INSPIRE].

[4] I. Bena, J. Polchinski and R. Roiban, Hidden symmetries of the AdS $S_{5} \times S^{5}$ superstring, Phys. Rev. D 69 (2004) 046002 [hep-th/0305116] [INSPIRE].

[5] J.A. Minahan and K. Zarembo, The Bethe ansatz for $\mathcal{N}=4$ super Yang-Mills, JHEP 03 (2003) 013 [hep-th/0212208] [INSPIRE].

[6] V.A. Kazakov, A. Marshakov, J.A. Minahan and K. Zarembo, Classical/quantum integrability in AdS/CFT, JHEP 05 (2004) 024 [hep-th/0402207] [INSPIRE].

[7] N. Beisert, V. Dippel and M. Staudacher, A novel long range spin chain and planar $\mathcal{N}=4$ super Yang-Mills, JHEP 07 (2004) 075 [hep-th/0405001] [INSPIRE]. 
[8] G. Arutyunov, S. Frolov and M. Staudacher, Bethe ansatz for quantum strings, JHEP 10 (2004) 016 [hep-th/0406256] [INSPIRE].

[9] N. Beisert and M. Staudacher, Long-range $\mathrm{psu}(2,2 \mid 4)$ Bethe ansätze for gauge theory and strings, Nucl. Phys. B 727 (2005) 1 [hep-th/0504190] [INSPIRE].

[10] N. Beisert, The SU(2|2) dynamic S-matrix, Adv. Theor. Math. Phys. 12 (2008) 945 [hep-th/0511082] [INSPIRE].

[11] R.A. Janik, The $A d S_{5} \times S^{5}$ superstring worldsheet S-matrix and crossing symmetry, Phys. Rev. D 73 (2006) 086006 [hep-th/0603038] [INSPIRE].

[12] N. Beisert, R. Hernandez and E. Lopez, A crossing-symmetric phase for $A d S_{5} \times S^{5}$ strings, JHEP 11 (2006) 070 [hep-th/0609044] [INSPIRE].

[13] N. Beisert, B. Eden and M. Staudacher, Transcendentality and crossing, J. Stat. Mech. (2007) P01021 [hep-th/0610251] [INSPIRE].

[14] G. Arutyunov and S. Frolov, String hypothesis for the $A d S_{5} \times S^{5}$ mirror, JHEP 03 (2009) 152 [arXiv:0901.1417] [INSPIRE].

[15] N. Gromov, V. Kazakov and P. Vieira, Exact spectrum of anomalous dimensions of planar $N=4$ supersymmetric Yang-Mills theory, Phys. Rev. Lett. 103 (2009) 131601 [arXiv:0901.3753] [INSPIRE].

[16] D. Bombardelli, D. Fioravanti and R. Tateo, Thermodynamic Bethe ansatz for planar AdS/CFT: a proposal, J. Phys. A 42 (2009) 375401 [arXiv:0902.3930] [InSPIRE].

[17] N. Gromov, V. Kazakov, A. Kozak and P. Vieira, Exact spectrum of anomalous dimensions of planar $N=4$ supersymmetric Yang-Mills theory: TBA and excited states, Lett. Math. Phys. 91 (2010) 265 [arXiv:0902 .4458] [INSPIRE].

[18] G. Arutyunov and S. Frolov, Thermodynamic Bethe ansatz for the $A d S_{5} \times S^{5}$ mirror model, JHEP 05 (2009) 068 [arXiv: 0903.0141] [INSPIRE].

[19] N. Gromov, V. Kazakov, S. Leurent and D. Volin, Quantum spectral curve for planar $\mathcal{N}=4$ super-Yang-Mills theory, Phys. Rev. Lett. 112 (2014) 011602 [arXiv:1305.1939] [INSPIRE].

[20] L.F. Alday, J.R. David, E. Gava and K.S. Narain, Structure constants of planar $\mathcal{N}=4$ Yang-Mills at one loop, JHEP 09 (2005) 070 [hep-th/0502186] [INSPIRE].

[21] L.F. Alday, J.R. David, E. Gava and K.S. Narain, Towards a string bit formulation of $\mathcal{N}=4$ super Yang-Mills, JHEP 04 (2006) 014 [hep-th/0510264] [INSPIRE].

[22] J. Escobedo, N. Gromov, A. Sever and P. Vieira, Tailoring three-point functions and integrability, JHEP 09 (2011) 028 [arXiv: 1012.2475] [INSPIRE].

[23] J. Escobedo, N. Gromov, A. Sever and P. Vieira, Tailoring three-point functions and integrability II. Weak/strong coupling match, JHEP 09 (2011) 029 [arXiv:1104.5501] [INSPIRE].

[24] N. Gromov, A. Sever and P. Vieira, Tailoring three-point functions and integrability III. Classical tunneling, JHEP 07 (2012) 044 [arXiv:1111.2349] [INSPIRE].

[25] N. Gromov and P. Vieira, Tailoring three-point functions and integrability IV. $\Theta$-morphism, JHEP 04 (2014) 068 [arXiv: 1205.5288] [INSPIRE].

[26] N. Gromov and P. Vieira, Quantum integrability for three-point functions of maximally supersymmetric Yang-Mills theory, Phys. Rev. Lett. 111 (2013) 211601 [arXiv:1202.4103] [INSPIRE]. 
[27] I. Kostov, Three-point function of semiclassical states at weak coupling, J. Phys. A 45 (2012) 494018 [arXiv:1205.4412] [INSPIRE].

[28] O. Foda, Y. Jiang, I. Kostov and D. Serban, A tree-level 3-point function in the SU(3)-sector of planar $\mathcal{N}=4 S Y M$, JHEP 10 (2013) 138 [arXiv:1302.3539] [INSPIRE].

[29] Y. Jiang, I. Kostov, F. Loebbert and D. Serban, Fixing the quantum three-point function, JHEP 04 (2014) 019 [arXiv:1401.0384] [INSPIRE].

[30] R.A. Janik and A. Wereszczynski, Correlation functions of three heavy operators: the AdS contribution, JHEP 12 (2011) 095 [arXiv: 1109.6262] [INSPIRE].

[31] Y. Kazama and S. Komatsu, On holographic three point functions for GKP strings from integrability, JHEP 01 (2012) 110 [Erratum ibid. 06 (2012) 150] [arXiv:1110.3949] [INSPIRE].

[32] Y. Kazama and S. Komatsu, Wave functions and correlation functions for GKP strings from integrability, JHEP 09 (2012) 022 [arXiv:1205.6060] [INSPIRE].

[33] Y. Kazama and S. Komatsu, Three-point functions in the SU(2) sector at strong coupling, JHEP 03 (2014) 052 [arXiv: 1312.3727] [INSPIRE].

[34] E.I. Buchbinder and A.A. Tseytlin, Semiclassical correlators of three states with large $S^{5}$ charges in string theory in $A d S_{5} \times S^{5}$, Phys. Rev. D 85 (2012) 026001 [arXiv:1110.5621] [INSPIRE].

[35] T. Klose and T. McLoughlin, A light-cone approach to three-point functions in $A d S_{5} \times S^{5}$, JHEP 04 (2012) 080 [arXiv:1106.0495] [INSPIRE].

[36] J.A. Minahan, Holographic three-point functions for short operators, JHEP 07 (2012) 187 [arXiv: 1206.3129] [INSPIRE].

[37] T. Bargheer, J.A. Minahan and R. Pereira, Computing three-point functions for short operators, JHEP 03 (2014) 096 [arXiv:1311.7461] [INSPIRE].

[38] K. Zarembo, Holographic three-point functions of semiclassical states, JHEP 09 (2010) 030 [arXiv: 1008.1059] [INSPIRE].

[39] M.S. Costa, R. Monteiro, J.E. Santos and D. Zoakos, On three-point correlation functions in the gauge/gravity duality, JHEP 11 (2010) 141 [arXiv:1008.1070] [INSPIRE].

[40] E.I. Buchbinder and A.A. Tseytlin, On semiclassical approximation for correlators of closed string vertex operators in AdS/CFT, JHEP 08 (2010) 057 [arXiv: 1005.4516] [INSPIRE].

[41] R. Roiban and A.A. Tseytlin, On semiclassical computation of 3-point functions of closed string vertex operators in $A d S_{5} \times S^{5}$, Phys. Rev. D 82 (2010) 106011 [arXiv:1008.4921] [INSPIRE].

[42] R. Hernandez, Three-point correlation functions from semiclassical circular strings, J. Phys. A 44 (2011) 085403 [arXiv:1011.0408] [inSPIRE].

[43] S. Ryang, Correlators of vertex operators for circular strings with winding numbers in $A d S_{5} \times S^{5}, J H E P 01$ (2011) 092 [arXiv: 1011.3573] [INSPIRE].

[44] G. Georgiou, Two and three-point correlators of operators dual to folded string solutions at strong coupling, JHEP 02 (2011) 046 [arXiv:1011.5181] [INSPIRE].

[45] J.G. Russo and A.A. Tseytlin, Large spin expansion of semiclassical 3-point correlators in $A d S_{5} \times S^{5}, J H E P 02$ (2011) 029 [arXiv: 1012.2760] [INSPIRE]. 
[46] C. Park and B.-H. Lee, Correlation functions of magnon and spike, Phys. Rev. D 83 (2011) 126004 [arXiv: 1012.3293] [InSPIRE].

[47] E.I. Buchbinder and A.A. Tseytlin, Semiclassical four-point functions in $A d S_{5} \times S^{5}$, JHEP 02 (2011) 072 [arXiv: 1012.3740] [INSPIRE].

[48] D. Bak, B. Chen and J.-B. Wu, Holographic correlation functions for open strings and branes, JHEP 06 (2011) 014 [arXiv: 1103.2024] [INSPIRE].

[49] A. Bissi, C. Kristjansen, D. Young and K. Zoubos, Holographic three-point functions of giant gravitons, JHEP 06 (2011) 085 [arXiv:1103.4079] [INSPIRE].

[50] D. Arnaudov, R.C. Rashkov and T. Vetsov, Three and four-point correlators of operators dual to folded string solutions in $A d S_{5} \times S^{5}$, Int. J. Mod. Phys. A 26 (2011) 3403 [arXiv:1103.6145] [INSPIRE].

[51] R. Hernandez, Three-point correlators for giant magnons, JHEP 05 (2011) 123 [arXiv:1104.1160] [INSPIRE].

[52] C. Ahn and P. Bozhilov, Three-point correlation functions of giant magnons with finite size, Phys. Lett. B 702 (2011) 286 [arXiv: 1105.3084] [INSPIRE].

[53] A. Bissi, T. Harmark and M. Orselli, Holographic 3-point function at one loop, JHEP 02 (2012) 133 [arXiv:1112.5075] [InSPIRE].

[54] P. Bozhilov, P. Furlan, V.B. Petkova and M. Stanishkov, On the semiclassical 3-point function in $A d S_{3}$, Phys. Rev. D 86 (2012) 066005 [arXiv:1204.1322] [INSPIRE].

[55] P. Caputa, R. de Mello Koch and K. Zoubos, Extremal versus non-extremal correlators with giant gravitons, JHEP 08 (2012) 143 [arXiv:1204.4172] [INSPIRE].

[56] J. Caetano and J. Toledo, $\chi$-systems for correlation functions, arXiv:1208.4548 [INSPIRE].

[57] H. Lin, Giant gravitons and correlators, JHEP 12 (2012) 011 [arXiv: 1209.6624] [INSPIRE].

[58] G. Grignani and A.V. Zayakin, Matching three-point functions of BMN operators at weak and strong coupling, JHEP 06 (2012) 142 [arXiv:1204.3096] [INSPIRE].

[59] G. Grignani and A.V. Zayakin, Three-point functions of BMN operators at weak and strong coupling II. One loop matching, JHEP 09 (2012) 087 [arXiv:1205.5279] [INSPIRE].

[60] W. Schulgin and A.V. Zayakin, Three-BMN correlation functions: integrability vs. string field theory. One-loop mismatch, JHEP 10 (2013) 053 [arXiv: 1305.3198] [INSPIRE].

[61] N.R. Constable et al., PP wave string interactions from perturbative Yang-Mills theory, JHEP 07 (2002) 017 [hep-th/0205089] [INSPIRE].

[62] M. Spradlin and A. Volovich, Superstring interactions in a pp-wave background, Phys. Rev. D 66 (2002) 086004 [hep-th/0204146] [INSPIRE].

[63] A. Pankiewicz and B. Stefański Jr., PP wave light cone superstring field theory, Nucl. Phys. B 657 (2003) 79 [hep-th/0210246] [InSPIRE].

[64] C.-S. Chu and V.V. Khoze, Correspondence between the three point BMN correlators and the three string vertex on the pp wave, JHEP 04 (2003) 014 [hep-th/0301036] [INSPIRE].

[65] S. Dobashi and T. Yoneya, Resolving the holography in the plane-wave limit of AdS/CFT correspondence, Nucl. Phys. B 711 (2005) 3 [hep-th/0406225] [INSPIRE].

[66] Z. Bajnok, The formfactor bootstrap, talk given at Nordita Workshop: Exact Results in Gauge-String Dualities, Nordita, Stockholm Sweden (2012). 
[67] T. Klose and T. McLoughlin, Worldsheet form factors in AdS/CFT, Phys. Rev. D 87 (2013) 026004 [arXiv: 1208. 2020] [InSPIRE].

[68] T. Klose and T. McLoughlin, Comments on world-sheet form factors in AdS/CFT, arXiv:1307.3506 [INSPIRE].

[69] L.D. Landau and E.M. Lifshitz, Quantum mechanics, Pergamon Press (1965).

[70] G. Arutyunov, S. Frolov and M. Zamaklar, Finite-size effects from giant magnons, Nucl. Phys. B 778 (2007) 1 [hep-th/0606126] [INSPIRE].

[71] R.A. Janik and T. Lukowski, Wrapping interactions at strong coupling: the giant magnon, Phys. Rev. D 76 (2007) 126008 [arXiv:0708.2208] [InSPIRE].

[72] M. Spradlin and A. Volovich, Dressing the giant magnon, JHEP 10 (2006) 012 [hep-th/0607009] [INSPIRE].

[73] C. Kalousios, G. Papathanasiou and A. Volovich, Exact solutions for N-magnon scattering, JHEP 08 (2008) 095 [arXiv: 0806. 2466] [INSPIRE].

[74] D.M. Hofman and J.M. Maldacena, Giant magnons, J. Phys. A 39 (2006) 13095 [hep-th/0604135] [INSPIRE].

[75] S. Britton and S. Frolov, Free field representation and form factors of the chiral Gross-Neveu model, JHEP 11 (2013) 076 [arXiv: 1305.6252] [INSPIRE].

[76] To appear.

[77] B. Pozsgay and G. Takács, Form factors in finite volume. II. Disconnected terms and finite temperature correlators, Nucl. Phys. B 788 (2008) 209 [arXiv:0706.3605] [INSPIRE].

[78] B. Pozsgay, Form factor approach to diagonal finite volume matrix elements in integrable QFT, JHEP 07 (2013) 157 [arXiv: 1305.3373] [INSPIRE]. 